\title{
TECHNICALLY NATURAL DARK ENERGY FROM LORENTZ BREAKING
}

\author{
D. Blas, ${ }^{a}$ S. Sibiryakov, \\ ${ }^{a}$ FSB/ITP/LPPC, École Polytechnique Fédérale de Lausanne, \\ CH-1015, Lausanne, Switzerland \\ ${ }^{b}$ Institute for Nuclear Research of the Russian Academy of Sciences, \\ 60th October Anniversary Prospect, 7a, 117312 Moscow, Russia
}

\begin{abstract}
We construct a model of dark energy with a technically natural small contribution to cosmic acceleration, i.e. this contribution does not receive corrections from other scales in the theory. The proposed acceleration mechanism appears generically in the low-energy limit of gravity theories with violation of Lorentz invariance that contain a derivatively coupled scalar field $\Theta$. The latter may be the Goldstone field of a broken global symmetry. The model, that we call $\Theta C D M$, is a valid effective field theory up to a high cutoff just a few orders of magnitude below the Planck scale. Furthermore, it can be ultraviolet-completed in the context of Hořava gravity. We discuss the observational predictions of the model. Even in the absence of a cosmological constant term, the expansion history of the Universe is essentially indistinguishable from that of $\Lambda$ CDM. The difference between the two theories appears at the level of cosmological perturbations. We find that in $\Theta C D M$ the matter power spectrum is enhanced at subhorizon scales compared to $\Lambda \mathrm{CDM}$. This property can be used to discriminate the model from $\Lambda \mathrm{CDM}$ with current cosmological data.
\end{abstract}




\section{Contents}

1 Introduction $\quad[2$

2 $\Theta \mathrm{CDM}$ model for cosmic acceleration

3 Preview of linear perturbations $\quad 10$

4 Linearized equations in FRW $\quad 14$

5 Cosmological perturbations: qualitative analysis $\quad 17$

5.1 Different regimes of evolution ...................... 18

5.2 Corrections to the metric perturbations . . . . . . . . . . . 25

6 Cosmological perturbations: numerical results $\quad 27$

\begin{tabular}{ll|l|}
7 & Conclusions & 32 \\
\hline
\end{tabular}

\begin{tabular}{|l|l|} 
A The numerical procedure & 34 \\
\hline
\end{tabular}

\section{Introduction}

Current cosmological observations indicate that the Universe recently entered a phase of accelerated expansion (see e.g. [1] and references therein). The driving force responsible for this phenomenon is normally called dark energy (DE) and its nature remains one of the major puzzles of contemporary physics. The standard explanation invokes a cosmological constant (CC), and the corresponding model - $\Lambda \mathrm{CDM}$ - manages to fit the data rather well [1]. However, on the theoretical side this explanation faces a serious problem. The measured value of the $\mathrm{CC}$ is many orders of magnitude smaller than the one expected on theoretical grounds. The latter is estimated as the value of quantum corrections to the vacuum energy plus the contribution related to possible phase transitions in the early Universe and is set by the energy scales of elementary particle interactions. This is the essence of the famous old Cosmological Constant Problem [2].

There exist two approaches to address this problem. The anthropic explanation assumes that the $\mathrm{CC}$ is indeed behind $\mathrm{DE}$ and claims that the observed value of the $\mathrm{CC}$ is small because the very existence of observers is possible only in a universe with small CC. The dynamical realization of this idea invokes the concept of a landscape of many vacua with 
different values of the potential energy and transitions between them. Thus the underlying theory must be necessarily very complex, and it is a matter of ongoing debate whether this picture possesses any predictive power [3, 4, [5, 6, 7].

An alternative to the anthropic reasoning is to declare that DE is not related to the CC. For this mechanism to work, one first needs to get rid of the CC contribution to acceleration. One may speculate that there is some deep (presumably nonperturbative) mechanism that reduces the value of the $\mathrm{CC}$, defined as the value of the total potential energy of all the fields at the absolute minimum, to zero [8, 9, 10]. In this picture the observed accelerated expansion of the Universe must either be produced by some dynamical component of the matter sector or be due to modification of the laws of gravity at the cosmological distances leading to theories of dynamical DE (sometimes referred to as quintessence). Many models of this type have been constructed, see [11] for a review. However, a lot of them suffer from one or a few of common problems (which are often just other faces of the CC problem). The simplest models of quintessence involve very light scalar fields with the mass smaller than the present Hubble parameter. Generically, these masses are unstable with respect to quantum corrections which reintroduces a fine-tuning problem. With the additional assumption of an approximate global shift symmetry of the scalar field (in other words, assuming that this field is a pseudo-Goldstone boson) its mass can be protected from perturbative corrections [12. However, the VEV that breaks the symmetry corresponding to the pseudo-Goldstone field is required to have the value of order or above the Planck scale, which is believed to be problematic when non-perturbative quantum gravity effects are taken into account [13]. Finally, from the purely observational perspective a drawback of this last class of theories is that, except for fine-tuned cases, their predictions are practically indistinguishable from those of $\Lambda$ CDM which makes impossible the discrimination of these theories from the CC. From this perspective, the models of DE involving non-linear potentials and/or kinetic terms for the fields and their interaction with dark and ordinary matter are more interesting due to their non-trivial phenomenology. However, the non-linear structure of the potentials makes these theories strongly coupled at a low cutoff scale (typically of order $10 \mathrm{eV}$ ). Thus, these models can be understood only as effective theories below this cutoff, raising the issue of a missing ultraviolet (UV) completion at low energy scales.

In this paper we present a simple model for dynamical DE that avoids the aforementioned problems. Assuming a vanishing CC, the smallness of the parameter setting the value of DE density in the model is protected by a discrete symmetry. The model is a valid effective field theory up to a very high scale just a few orders below the Planck mass. Remarkably, 
above this scale the model admits a UV completion in the form of the Hořava's model [14] (more precisely, its consistent non-projectable extension [15, 16, 17]) which is a candidate for a renormalizable theory of quantum gravity. Finally, while the expansion history of the Universe in our model is basically indistinguishable from that of $\Lambda \mathrm{CDM}$, the growth of cosmological perturbations is different, which can allow to discriminate this model from $\Lambda \mathrm{CDM}$ using the structure formation and weak lensing data 1 .

The price to pay for these nice features is to admit violation of Lorentz symmetry in the gravity sector. This possibility is interesting on its own right and has received a great deal of attention recently, the prototypical models describing the possible patterns of Lorentz symmetry breaking being the Einstein-aether [19] and the ghost condensate [20] theories. A major issue in this kind of theories is the possible transmission of Lorentz breaking to the fields of the Standard Model (SM) which would be unacceptable as Lorentz invariance is tested to a very high precision in the SM sector [21]. Nevertheless, this problem is completely unrelated to DE and may be addressed using some dynamical mechanisms [22, 23, 24]. Once the emergence of Lorentz invariance at low energies for the SM is achieved, there is no extra requirement to make our proposal for DE technically natural.

It is worth mentioning another interesting property of the model we are going to present that may shed some light on the old CC problem (vanishing of the vacuum energy). In the absence of a $\mathrm{CC}$ and matter (including dark matter) it admits two solutions, corresponding to Minkowski and de Sitter space-times. The latter solution is stable while the former is unstable with respect to long-wavelength perturbations. For that reason, the Minkowski solution is never reached during the cosmological expansion and instead the system approaches the de Sitter branch. In this sense the cosmology of our model is self-accelerating (cf. [25]). The instability of the Minkowski vacuum is a purely infrared phenomenon and is cut off at certain finite momentum. In other words, the Minkowski vacuum is perfectly well-defined from the point of view of the UV physics. This is consistent with the previous idea that the existence of such a vacuum may be required by some unknown principle which assures the cancellation of CC. In this paper we will not pursue the discussion about what kind of principle this could be.

This paper is organized as follows. In Sec. 2] we present the model and describe the cosmological solutions. In Sec. 3 we make a preliminary analysis of perturbations in the expanding and Minkowski backgrounds and show that in the latter case long-wavelength modes are

\footnotetext{
${ }^{1} \mathrm{~A}$ non-trivial feature of the model is parametric enhancement of the signal in the structure formation that allows to have sizable effect even after satisfying stringent Solar system constraints.
} 
unstable. In Sec. 4 we turn to the detailed analysis of the cosmological perturbations in the Friedmann - Robertson - Walker (FRW) universe and derive the set of linearized equations. These equations are solved analytically in Sec. 5 in various approximations. The reader who is interested in the final results can skip this section and go directly to Sec. 6 where we report the results of the numerical integration of the linearized equations. Section 7 is devoted to conclusions. Appendix A contains some details about our numerical procedure.

\section{$2 \quad \Theta C D M$ model for cosmic acceleration}

We start by considering theories where local Lorentz invariance is broken by the presence of a unit time-like vector $u_{\mu}$. We will restrict to the case where the vector is orthogonal to a set of space-like 3-dimensional surfaces that foliate the space-time. Parameterizing these surfaces as the constant levels of a function $\varphi(x), u_{\mu}$ can be written in the form 2 ,

$$
u_{\mu}=\frac{\partial_{\mu} \varphi}{\sqrt{g^{\nu \rho} \partial_{\nu} \varphi \partial_{\rho} \varphi}} .
$$

Note that $u_{\mu}$ is invariant under reparametrizations

$$
\varphi \mapsto \tilde{\varphi}=f(\varphi)
$$

for an arbitrary monotonic function $f$. The most general action describing the coupling of $u_{\mu}$ to gravity and containing a total of two derivatives acting on $u_{\mu}$ has the form 3 ,

$$
S_{[E H \chi]}=-\frac{M_{0}^{2}}{2} \int \mathrm{d}^{4} x \sqrt{-g}\left(R+K_{\sigma \rho}^{\mu \nu} \nabla_{\mu} u^{\sigma} \nabla_{\nu} u^{\rho}\right)
$$

where $R$ is the space-time curvature, $M_{0}$ is a mass parameter related to the Planck mass and

$$
K_{\sigma \rho}^{\mu \nu}=\beta \delta_{\rho}^{\mu} \delta_{\sigma}^{\nu}+\lambda \delta_{\sigma}^{\mu} \delta_{\rho}^{\nu}+\alpha u^{\mu} u^{\nu} g_{\sigma \rho},
$$

with $\alpha, \beta, \lambda$ free dimensionless parameters. This model has been proposed in [17] (see also [18]) and was dubbed 'khrono-metric' because it introduces the notion of a preferred global time coordinate set by the field $\varphi(x)$ - the 'khronon' field. As discussed in [17], Eq. (3) describes the low-energy limit of the consistent extension [15, 16] of Hořava gravity [14].

\footnotetext{
${ }^{2}$ Our convention for the metric signature is $(+,-,-,-)$.

${ }^{3}$ We use subindices inside square brackets to distinguish the quantities referring to the different sectors of the model.

${ }^{4}$ Note that the parameter $\lambda$ in (3) corresponds to $\lambda^{\prime}$ in the notations of [17.
} 
The latter has been proposed as a power-counting renormalizable model of quantum gravity. In the case when the parameters $\alpha, \beta, \lambda$ are of the same order the action (3) gives a valid effective field theory up to the scale $\Lambda_{\text {cutoff }}=\min \left\{M_{0} \sqrt{\alpha}, M_{0}\right\}$. Note that when written in terms of the khronon field $\varphi$ the action (3) contains four derivatives and thus gives rise to a fourth order equation of motion. However, the structure of the higher derivative terms is such that, for a proper choice of the time variable, only two of these derivatives are temporal [17. Thus the khronon field contains only one propagating degree of freedom. In particular, no instabilities, that are in general associated with higher derivative Lagrangians, arise.

Experimental data impose constraints on the parameters $\alpha, \beta, \lambda$. For general order one ratios between these constants the strongest bounds come from the constraints on the parameters $\alpha_{1}^{P P N}, \alpha_{2}^{P P N}$ of the parametrized post-Newtonian (PPN) formalism 5 and require $\alpha, \beta, \lambda$ to be smaller than $10^{-6}$ [17]. However, for the special case $\alpha=2 \beta$ both $\alpha_{1}^{P P N}, \alpha_{2}^{P P N}$ vanish and one is left with a much weaker bound $\alpha, \beta, \lambda \lesssim 0.1$ coming from the dynamics of the Big Bang Nucleosynthesis (BBN) [27] and the emission of gravitational waves in binary systems [28]. Another special case is $\beta=0, \alpha=\lambda$. Then the PPN parameter $\alpha_{2}^{P P N}$ vanishes, while the constraint on $\alpha_{1}^{P P N}$ gives $\alpha, \lambda<10^{-4}$.

If we do not insist on the existence of a known UV-completion and are content with an effective theory with high cutoff, we can relax the condition (11) and consider a general unit time-like vector. This would lead us to the Einstein-aether theory [19] (see [29] for a review). The action for the general aether has the same form as (3) with an extra term6. Physically, the Einstein-aether model differs from the khrono-metric theory by the presence of transverse vector modes among the perturbations. However, these modes do not affect the homogeneous cosmological expansion, nor the linear evolution of scalar cosmological perturbation] Thus, the analysis of the present paper applies without changes to the general aether model. Just for clarity we concentrate on the hypersurface-orthogonal case corresponding to the khrono-metric theory.

To the system (3) we add a scalar field $\Theta$ with the symmetry under the shifts

$$
\Theta \mapsto \Theta+\text { const . }
$$

The precise origin of this field is not important for our purposes. However, an example that is useful to keep in mind is a Goldstone boson corresponding to a spontaneously broken

\footnotetext{
${ }^{5}$ These PPN parameters describe effects of local Lorentz violation and are zero in general relativity. The experimental bounds are [26]: $\left|\alpha_{1}^{P P N}\right| \lesssim 10^{-4},\left|\alpha_{2}^{P P N}\right| \lesssim 10^{-7}$.

${ }^{6}$ For a hypersurface-orthogonal aether this term can be written as a combination of those present in (3).

${ }^{7}$ The transverse modes can lead to interesting effects in the CMB polarization 30.
} 
global symmetry 8 . For this reason, we will refer to the field $\Theta$ as "Goldstone field" in the rest of the paper. In contrast to the pseudo-Goldstone quintessence models [12, we assume the symmetry (5) to be exact. The interaction between the Goldstone and the khronon must be invariant under (2) and (5). Keeping only the operators that have dimensions up to 4 and thus are relevant for physics at long distances, we obtain the following general action 9 for $\Theta$ :

$$
S_{[\Theta]}=\int \mathrm{d}^{4} x \sqrt{-g}\left(\frac{g^{\mu \nu} \partial_{\mu} \Theta \partial_{\nu} \Theta}{2}+\varkappa \frac{\left(u^{\mu} \partial_{\mu} \Theta\right)^{2}}{2}+\mu^{2} u^{\mu} \partial_{\mu} \Theta\right) .
$$

Here the coupling constant $\varkappa$ is dimensionless, while $\mu$ has dimension of mass. It is important to notice that the $\mu$-term is the only one that is not invariant under $\Theta \mapsto-\Theta$. Thus, assuming that the UV completion of the theory respects this discrete symmetry, the value of $\mu$ is stable under radiative corrections.

The physical effect of the two khronon-Goldstone couplings in (6) is quite different. Let us consider the dynamics of the Goldstone field in the background with flat metric and $u_{\mu}=(1,0,0,0)$. Then the third term in (6) becomes a total derivative and does not affect the dynamics, while the second term modifies the propagation velocity $c_{\Theta}$ of the Goldstone field:

$$
c_{\Theta}^{2}=\frac{1}{1+\varkappa}
$$

We will see in a moment that the effects of the khronon-Goldstone coupling described by the third term become essential in curved backgrounds and modify the dynamics of both fields at cosmological scales. A coupling of this form was introduced in [31] in the context of inflation in the Einstein-aether model. A similar coupling was also considered in [32].

We will study the cosmological evolution in the model with the action

$$
S=S_{[E H \chi]}+S_{[\Theta]}+S_{[m]}
$$

\footnotetext{
${ }^{8}$ Note that the scale of this putative symmetry breaking will be completely irrelevant for the analysis in this paper (provided that it is higher than the temperature of the universe at the epochs of interest, which is roughly a few eV). In particular, it can be safely below the Planck mass.

${ }^{9}$ One could consider including in the action operators

$$
u^{\mu} \nabla_{\mu} u^{\nu} \partial_{\nu} \Theta, \quad\left(\nabla_{\mu} u^{\mu}\right) u^{\nu} \partial_{\nu} \Theta
$$

that formally have dimension 3. We omit them for two reasons. First, these operators can be forbidden by imposing the symmetry under simultaneous change of the signs of $\Theta$ and $u^{\mu}: \Theta \mapsto-\Theta, u^{\mu} \mapsto-u^{\mu}$. More importantly, these operators actually have dimension higher than 4 when expressed in terms of the canonically normalized khronon perturbations, and thus do not affect the long distance physics.
} 
where $S_{[E H \chi]}$ and $S_{[\Theta]}$ have been introduced above and $S_{[m]}$ stands for the action of matter, which includes baryonic matter, dark matter and radiation. We assume that $S_{[m]}$ has the standard form with matter interacting minimally with the metric $g_{\mu \nu}$. In particular, we assume that there are no direct couplings between the matter sector and the khronon or Goldstone fields10. We will refer to this model as $\Theta C D M$.

Introducing the Ansatz for the most general spatially homogeneous solution

$$
\begin{gathered}
\mathrm{d} s^{2}=N^{2}(t) \mathrm{d} t^{2}-a^{2}(t) \delta_{i j} \mathrm{~d} x^{i} \mathrm{~d} x^{j} \\
\varphi=\varphi(t), \quad \Theta=\Theta(t)
\end{gathered}
$$

into the actions (3), (6) we obtain

$$
S_{[E H \chi]}+S_{[\Theta]}=-\frac{M_{0}^{2}(6+3 \beta+9 \lambda)}{2} \int \mathrm{d}^{4} x \frac{\dot{a}^{2} a}{N}+\int \mathrm{d}^{4} x\left(\frac{a^{3}}{2 N c_{\Theta}^{2}} \dot{\Theta}^{2}+\mu^{2} a^{3} \dot{\Theta}\right) .
$$

Note that the field $\varphi$ has dropped out of the action, which is a consequence of the invariance under (2). Varying the action with respect to $\Theta$ and $N$ we obtain the cosmological equations,

$$
\begin{aligned}
& \frac{\mathrm{d}}{\mathrm{d} t}\left(\frac{a^{3}}{N c_{\Theta}^{2}} \dot{\Theta}+\mu^{2} a^{3}\right)=0, \\
& H^{2}=\frac{8 \pi G_{\cos m}}{3}\left(\frac{\dot{\Theta}^{2}}{2 N^{2} c_{\Theta}^{2}}+\rho_{m}\right),
\end{aligned}
$$

where $H \equiv \dot{a} /(a N)$ is the Hubble rate and

$$
G_{\text {cosm }}=\frac{1}{8 \pi M_{0}^{2}(1+\beta / 2+3 \lambda / 2)}
$$

On the r.h.s. of the Friedmann equation (10) we added the contribution $\rho_{m}$ of ordinary matter. Note that the expression for the gravitational constant $G_{\text {cosm }}$ entering the Friedmann equation is corrected by the presence of the khronon compared to the standard GR formula

\footnotetext{
${ }^{10}$ This must be considered as a simplifying assumption. For the ordinary matter the direct couplings to the khronon field would imply violation of the Lorentz symmetry within the SM sector and is tightly constrained by the experimental data. Once these constraints are satisfied, the effects in cosmology are negligible. On the other hand, for dark matter we do not have such strong observational evidence of Lorentz invariance. Allowing for direct coupling between dark matter and the khronon may lead to interesting effects that will be reported elsewhere [33. As for the coupling of SM / dark matter to the Goldstone, due to the symmetry (5) it must involve derivatives. Apart from possible kinetic mixing with a scalar gauge singlet that may be part of dark matter, this would produce higher order operators irrelevant for long-distance physics.
} 
$G=\left(8 \pi M_{0}^{2}\right)^{-1}$. As discussed in [15, 17], this differs from the expression for the gravitational coupling

$$
G_{N}=\frac{1}{8 \pi M_{0}^{2}(1-\alpha / 2)}
$$

appearing in the Newtonian potential of a localized source. The bound [27] $\left|G_{\text {cosm }} / G_{N}-1\right| \leq$ 0.13 from $\mathrm{BBN}$ thus requires the parameters $\alpha, \beta, \lambda$ to be smaller than 0.1 .

The equation (9) is easily solved with the result

$$
\frac{\dot{\Theta}}{N}=-\left(\mu^{2} c_{\Theta}^{2}+\frac{C}{a^{3}}\right),
$$

where $C$ is an integration constant. Due to the cosmological expansion the ratio $\dot{\Theta} / N$ is attracted to the constant non-zero value $-\mu^{2} c_{\Theta}^{2}$. On this attractor solution the field $\Theta$ produces the same contribution into the Friedmann equation (10) as the vacuum energy density

$$
V_{0, e f f}=\mu^{4} c_{\Theta}^{2} / 2
$$

In this way the model leads to accelerated cosmology even in the absence of the true cosmological constant. Due to the stability of the parameter $\mu$ under radiative corrections it is technically natural for the effective vacuum energy density (12) to be small.

Notice that the general solution (11), once introduced in the Friedmann equation (10), also produces a contribution scaling like $a^{-3}$, that one may be tempted to interpret as dark matter. However, this contribution is always accompanied by the stiff term proportional to $C^{2} a^{-6}$ that dominates over the dark matter-type contribution. To comply with the experimental constraints the stiff term must be small. We will set $C=0$ in what follows. Thus the $\Theta$-field cannot be responsible for dark matter, which one has to add as a separate component. This will be included in the part of the theory described by $S_{[m]}$ in (17).

It is worth stressing that we do not present any mechanism that could lead to vanishing of the vacuum energy and thus do not pretend to solve the old CC problem. However, we point out the following interesting property of the $\Theta C D M$ model that may shed some light on the resolution of this problem. In the absence of matter, $\rho_{m}=0$, the system of equations (9), (10) possesses a static solution with flat metric,

$$
N=a=\text { const }, \quad \dot{\Theta}=0 .
$$

However, this solution is never achieved if the evolution starts from a configuration with $\rho_{m} \neq 0$. Moreover, in the next section we will see that the Minkowski solution is unstable 
with respect to long-wavelength perturbations. On the other hand, at small scales the instability is cut off and from the viewpoint of the short distance physics (13) is a valid background. Thus one may hope to find a mechanism that would neutralize the CC in this background and ensure the existence of a (unstable) Minkowski vacuum. However, the dynamics drives the universe not to Minkowski but to the de Sitter attractor.

\section{Preview of linear perturbations}

As we saw in the previous section, at the level of homogeneous cosmology the attractor solution in the $\Theta C D M$ is indistinguishable from $\Lambda \mathrm{CDM}$. Then it is natural to wonder if the model leaves any distinctive signatures at the next level of approximation, namely in the evolution of linear cosmological perturbations. The analysis of the linearized perturbations is also indispensable to verify the stability of the accelerated solution.

Before going to the complete analysis which we postpone to the subsequent sections, let us consider a simplified setup where one neglects back-reaction of the khronon and Goldstone perturbations on the geometry. Formally this corresponds to taking the limit $M_{0} \rightarrow \infty$. As will be clear later, we must at the same time keep the scale

$$
M_{\alpha} \equiv \sqrt{\alpha} M_{0}
$$

fixed. This implies that in the limit of interest $\alpha \rightarrow 0$. We also assume that the ratios $\beta / \alpha$ and $\lambda / \alpha$ are fixed and of order one in this limit.

We start with the analysis of perturbations in Minkowski space-time. One writes

$$
\begin{aligned}
& \varphi(t, \mathbf{x})=\bar{\varphi}(t)+\chi(t, \mathbf{x}), \\
& \Theta(t, \mathbf{x})=\bar{\Theta}(t)+\xi(t, \mathbf{x}),
\end{aligned}
$$

where $\bar{\varphi}(t), \bar{\Theta}(t)$ are the background values of the fields and $\chi, \xi$ are the perturbations. Note that due to the reparametrization symmetry (2) we can always set

$$
\bar{\varphi}(t)=t
$$

Also for the Minkowski solution $\bar{\Theta}(t)=$ const. Expanding the actions (3) , (6) to quadratic order in fluctuations we obtain

$$
\left[S_{[E H \chi]}+S_{[\Theta]}\right]^{(2)}=\int \mathrm{d}^{4} x\left[\frac{M_{\alpha}^{2}}{2}\left(\partial_{i} \dot{\chi}\right)^{2}-\frac{M_{\alpha}^{2} c_{\chi}^{2}}{2}(\Delta \chi)^{2}+\frac{\dot{\xi}^{2}}{2 c_{\Theta}^{2}}-\frac{\left(\partial_{i} \xi\right)^{2}}{2}-\mu^{2} \partial_{i} \chi \partial_{i} \xi\right]
$$


where $\Delta \equiv \partial_{i} \partial_{i}$ is the spatial Laplacian, and we have introduced the notation

$$
c_{\chi}^{2}=\frac{\beta+\lambda}{\alpha}
$$

Throughout the paper we will assume that $c_{\chi}^{2}$, as well as $c_{\Theta}^{2}$, are of order one. The action (17) yields the equations of motion,

$$
\begin{aligned}
& \Delta\left(\ddot{\chi}-c_{\chi}^{2} \Delta \chi+\frac{\mu^{2}}{M_{\alpha}^{2}} \xi\right)=0, \\
& \frac{\ddot{\xi}}{c_{\Theta}^{2}}-\Delta \xi-\mu^{2} \Delta \chi=0 .
\end{aligned}
$$

The overall Laplacian in the first equation can be cancelled out for any inhomogeneous configuration. Performing the Fourier decomposition

$$
\chi, \xi \propto \mathrm{e}^{-i \omega t+i \mathbf{k x}}
$$

we find the dispersion relations of the propagating modes,

$$
\omega_{ \pm}^{2}=\frac{1}{2}\left[\left(c_{\chi}^{2}+c_{\Theta}^{2}\right) k^{2} \pm \sqrt{\left(c_{\chi}^{2}-c_{\Theta}^{2}\right)^{2} k^{4}+\frac{4 \mu^{4} c_{\Theta}^{2}}{M_{\alpha}^{2}} k^{2}}\right] .
$$

This expression simplifies in two regimes. At $k$ larger than the critical momentum

$$
k_{c} \equiv \mu^{2} / M_{\alpha}
$$

the fields $\chi$ and $\xi$ decouple and describe two modes with linear dispersion relations

$$
\begin{aligned}
& \omega_{\chi}^{2}=c_{\chi}^{2} k^{2}, \\
& \omega_{\xi}^{2}=c_{\Theta}^{2} k^{2} .
\end{aligned}
$$

Note that both modes are stable. In the opposite case $k \ll k_{c}$ Eq. (21) takes the form,

$$
\omega_{ \pm}^{2}= \pm c_{\Theta} k_{c} k
$$

This dispersion relation is non-analytic: the frequency is proportional to the square root of the momentum. Clearly, one of the modes has purely imaginary frequency and thus exponentially grows with time. This signals instability of the Minkowski background with respect to long-wavelength perturbations. As we are going to see, this instability disappears in the expanding universe. 
As the next exercise we consider the evolution of the $\chi^{-}, \xi$-perturbations in an external FRW metric. We will work in the conformal time which corresponds to setting $N=a$ in (8a), and consider a general time-dependence of the scale factor. We expand again as in (15) $)$ where we fix (16). The background configuration $\bar{\Theta}(t)$ satisfies Eq. (11). To simplify the calculations we concentrate on the attractor solution

$$
\dot{\bar{\Theta}}=-a \mu^{2} c_{\Theta}^{2}
$$

After a straightforward computation we find the quadratic action

$$
\begin{aligned}
{\left[S_{[E H \chi]}+S_{[\Theta]}{ }^{(2)}=\int \mathrm{d}^{4} x a^{2}[\right.} & \frac{M_{\alpha}^{2}}{2}\left(\partial_{i} \dot{\chi}\right)^{2}-\frac{M_{\alpha}^{2} c_{\chi}^{2}}{2}(\Delta \chi)^{2} \\
& -\left(M_{\alpha}^{2} \dot{\mathcal{H}}(1-B)+M_{\alpha}^{2} \mathcal{H}^{2}(1+B)+a^{2} \mu^{4} c_{\Theta}^{4}\right) \frac{\left(\partial_{i} \chi\right)^{2}}{2} \\
& \left.+\frac{\dot{\xi}^{2}}{2 c_{\Theta}^{2}}-\frac{\left(\partial_{i} \xi\right)^{2}}{2}-a \mu^{2} c_{\Theta}^{2} \partial_{i} \chi \partial_{i} \xi\right]
\end{aligned}
$$

where we introduced the notations

$$
\begin{aligned}
& B=\frac{\beta+3 \lambda}{\alpha}, \\
& \mathcal{H} \equiv \frac{\dot{a}}{a}=H a,
\end{aligned}
$$

Note the appearance of a term with two space derivatives of $\chi$ which was absent in the case of Minkowski background. The action (26) yields the following equations,

$$
\begin{aligned}
& \ddot{\chi}+2 \mathcal{H} \dot{\chi}-c_{\chi}^{2} \Delta \chi+\left[\dot{\mathcal{H}}(1-B)+\mathcal{H}^{2}(1+B)+a^{2} c_{\Theta}^{4} k_{c}^{2}\right] \chi+\frac{a c_{\Theta}^{2} k_{c}^{2}}{\mu^{2}} \xi=0, \\
& \ddot{\xi}+2 \mathcal{H} \dot{\xi}-c_{\Theta}^{2} \Delta \xi-a \mu^{2} c_{\Theta}^{4} \Delta \chi=0,
\end{aligned}
$$

where in the first equation we have cancelled the overall Laplacian. The crucial difference from Eqs. (19) in the Minkowski case is the presence of an effective mass term for the field $\chi$,

$$
m_{\text {eff }}^{2}=\frac{\dot{\mathcal{H}}(1-B)+\mathcal{H}^{2}(1+B)}{a^{2}}+c_{\Theta}^{4} k_{c}^{2} .
$$

This expression deserves two comments. First, during the epoch of primordial inflation one can neglect the last term in (30) and also use $\dot{\mathcal{H}} \approx \mathcal{H}^{2}$. This gives $m_{\text {eff }}^{2} \approx 2 H_{\text {inf }}^{2}$, i.e. the effective khronon mass is of order the Hubble parameter at inflation and coincides with the effective mass of a conformally coupled scalar field. As a consequence, the khronon 
perturbations are not generated during inflation. This agrees with the result of [34] that no isocurvature perturbations associated with the khronon are produced during inflation.

Second, for certain choices of parameters the effective mass square (30) can become negative during the radiation and matter dominated epochs. This will lead to the growth of the khronon perturbations with wavelengths larger than the horizon size at the corresponding epoch. It is worth stressing that this growth is under control, its rate being proportional to the Hubble parameter. Even if this regime is definitely interesting from the phenomenological point of view, it corresponds to a rather special corner of the parameter space. In the present work we focus on the generic stable case.

Let us now analyze the behavior of subhorizon modes with large momenta and frequencies, $k, \omega \gg \mathcal{H}$. In this case we can neglect all $\mathcal{H}$-dependent terms in Eqs. (29) and perform the Fourier decomposition (20). However, the presence of a non-trivial background $\dot{\bar{\Theta}}$ still modifies the equations for the perturbations, eliminating any instability for the momenta inside the horizon. Explicitly, the dispersion relations are 11

$$
\omega_{ \pm}^{2}=\frac{1}{2}\left[\left(c_{\chi}^{2}+c_{\Theta}^{2}\right) k^{2}+c_{\Theta}^{4} k_{c}^{2} \pm \sqrt{\left(\left(c_{\chi}^{2}+c_{\Theta}^{2}\right) k^{2}+c_{\Theta}^{4} k_{c}^{2}\right)^{2}-4 c_{\chi}^{2} c_{\Theta}^{2} k^{4}}\right]
$$

For large momenta $k \gg k_{c}$ one recovers the linear dispersion relations (23) $)$. While at $k \ll k_{c}$ we have

$$
\begin{aligned}
& \omega_{+}^{2}=c_{\Theta}^{4} k_{c}^{2}+\left(c_{\chi}^{2}+c_{\Theta}^{2}\right) k^{2}, \\
& \omega_{-}^{2}=\frac{c_{\chi}^{2} k^{4}}{c_{\Theta}^{2} k_{c}^{2}} .
\end{aligned}
$$

We see that, in contrast to the case of Minkowski, both modes are stable (cf. (24)). One of them possesses a frequency gap, while the other has a dispersion relation with quadratic dependence of the frequency on momentum. This implies that the latter mode has low propagation velocity $c_{-} \sim k / k_{c} \ll 1$ and one expects it to cluster in the gravitational potential wells enhancing the growth of the cosmological perturbations. We are going to see below that this expectation is correct.

It is worth stressing that the analysis of this, as well as the subsequent sections, applies without change to the longitudinal sector of linear perturbations in the case when the khronometric model is substituted by the Einstein-aether theory to describe the Lorentz breaking.

\footnotetext{
${ }^{11}$ Here $\omega$ and $k$ should be understood as physical frequency and momentum which corresponds to putting $a=1$ in the formulas.
} 


\section{Linearized equations in FRW}

We now turn to the derivation of linearized equations of motion for $\Theta C D M$ including gravitational perturbations and matter fields. We start with the Einstein's equations

$$
R_{\mu \nu}-\frac{1}{2} g_{\mu \nu} R=\frac{1}{M_{0}^{2}}\left(T_{[\chi] \mu \nu}+T_{[\Theta] \mu \nu}+T_{[m] \mu \nu}\right)
$$

where $T_{[\chi] \mu \nu}, T_{[\Theta] \mu \nu}$ and $T_{[m] \mu \nu}$ are respectively the energy-momentum tensors of the khronon, Goldstone field and matter. They are defined as the variation of the corresponding action with respect to the metric,

$$
T_{[i] \mu \nu}=\frac{2}{\sqrt{-g}} \frac{\delta S_{[i]}}{\delta g^{\mu \nu}} .
$$

After a long but straightforward computation we obtain

$$
\begin{aligned}
& M_{0}^{-2} T_{[\chi] \mu \nu}=-\nabla_{\lambda} K_{\rho}^{\lambda} u^{\rho} u_{\mu} u_{\nu}+2 \nabla_{\lambda} K_{(\mu}^{\lambda} u_{\nu)}-\nabla_{\lambda}\left(K_{(\mu}^{\lambda} u_{\nu)}\right)-\nabla_{\lambda}\left(K_{(\mu \nu)} u^{\lambda}\right) \\
& +\nabla_{\lambda}\left(K_{(\mu}^{\lambda} u_{\nu)}\right)+\alpha\left[a_{\lambda} a^{\lambda} u_{\mu} u_{\nu}-2 a^{\lambda} \nabla_{(\mu} u_{\lambda} u_{\nu)}+a_{\mu} a_{\nu}\right]+\frac{1}{2} K_{\rho}^{\lambda} \nabla_{\lambda} u^{\rho} g_{\mu \nu}
\end{aligned}
$$

where

$$
\begin{gathered}
a_{\mu}=u^{\lambda} \nabla_{\lambda} u_{\mu}, \\
K_{\nu}^{\mu}=K_{\rho \nu}^{\lambda \mu} \nabla_{\lambda} u^{\rho},
\end{gathered}
$$

and the round brackets denote symmetrization of indices:

$$
K_{(\mu \nu)}=\frac{1}{2}\left(K_{\mu \nu}+K_{\nu \mu}\right) .
$$

Similarly, for $T_{[\Theta] \mu \nu}$ we find,

$$
\begin{aligned}
T_{[\Theta] \mu \nu}=\partial_{\mu} \Theta \partial_{\nu} \Theta+2\left(\varkappa u^{\lambda} \partial_{\lambda} \Theta\right. & \left.+\mu^{2}\right) u_{(\mu} \partial_{\nu)} \Theta-u_{\mu} u_{\nu}\left(\varkappa\left(u^{\lambda} \partial_{\lambda} \Theta\right)^{2}+\mu^{2} u^{\lambda} \partial_{\lambda} \Theta\right) \\
& -\left(\frac{\nabla^{\lambda} \Theta \partial_{\lambda} \Theta}{2}+\frac{\varkappa}{2}\left(u^{\lambda} \partial_{\lambda} \Theta\right)^{2}+\mu^{2} u^{\lambda} \partial_{\lambda} \Theta\right) g_{\mu \nu} .
\end{aligned}
$$

Finally, for the matter sector we will include only two decoupled components corresponding to cold matter $(\mathrm{cm})$ and radiation $(\gamma)$ in the perfect fluid approximation. Reducing the matter sector to this form is, of course, a crude simplification but it is convenient for illustrating the difference between $\Theta C D M$ and $\Lambda$ CDM. In this respect, whenever we refer to the $\Lambda \mathrm{CDM}$ model in the future, we will have in mind the case of GR with a CC and the previous matter content. We leave more accurate analysis of the cosmological perturbations for 
future research. Thus, for the energy-momentum tensor we take the standard hydrodynamic expression for each component

$$
T_{[m] \mu \nu}=\left(\rho_{[\gamma]}+p_{[\gamma]}\right) v_{[\gamma] \mu} v_{[\gamma] \nu}-p_{[\gamma]} g_{\mu \nu}+\left(\rho_{[\mathrm{cm}]}+p_{[\mathrm{cm}]}\right) v_{[\mathrm{cm}] \mu} v_{[\mathrm{cm}] \nu},
$$

where $\rho_{[a]}, p_{[a]}$ are the energy density and pressure of the different matter components and $v_{[a] \mu}$ are their 4-velocities.

To form a closed system the Einstein's equations must be supplemented by the equations of motion for the khronon, Goldstone field and matter. The first two of these equations are obtained by varying the sum of the actions (3) and (6) with respect to $\varphi$ and $\Theta$. This yields,

$$
\begin{aligned}
\nabla_{\rho}\left[\frac{P^{\rho \mu}}{\sqrt{X}}\left(-\nabla_{\nu} K^{\nu}{ }_{\mu}+\alpha a_{\nu} \nabla_{\mu} u^{\nu}-\frac{1}{M_{0}^{2}} \partial_{\mu} \Theta\left(\varkappa u^{\lambda} \partial_{\lambda} \Theta+\mu^{2}\right)\right)\right] & =0, \\
\square \Theta+\varkappa \nabla_{\mu}\left(u^{\mu} u^{\nu} \partial_{\nu} \Theta\right)+\mu^{2} \nabla_{\mu} u^{\mu} & =0,
\end{aligned}
$$

where

$$
\begin{gathered}
P^{\mu \nu}=g^{\mu \nu}-u^{\mu} u^{\nu}, \\
X=g^{\mu \nu} \partial_{\mu} \varphi \partial_{\nu} \varphi, \\
\square=g^{\mu \nu} \partial_{\mu} \partial_{\nu} .
\end{gathered}
$$

The equations of motion for matter follow from the conservation of the energy-momentum tensor of each component (recall that we assume the two components to be decoupled),

$$
\nabla^{\mu} T_{[\gamma] \mu \nu}=\nabla^{\mu} T_{[c m] \mu \nu}=0 .
$$

In fact, only one of the equations in (39) is an independent equation, as the conservation of the total matter energy-momentum tensor follows from the Einstein's equations and (38).

We now expand the above expressions to linear order in perturbations around a FRW background. The dynamics differ from the standard case only in the scalar sector of the perturbations and we concentrate on this in what follows. For the khronon and Goldstone fields we take the representation (15) with the background values satisfying (16), (25). The metric perturbations are chosen in the conformal Newton's gauge

$$
\mathrm{d} s^{2}=a^{2}(t)\left[(1+2 \phi) \mathrm{d} t^{2}-(1-2 \psi) \delta_{i j} \mathrm{~d} x^{i} \mathrm{~d} x^{j}\right] .
$$

Finally, for the scalar sector of the matter perturbations we write

$$
\rho_{[a]}=\bar{\rho}_{[a]}(t)+\delta \rho_{[a]}, \quad p_{[a]}=\bar{p}_{[a]}(t)+\delta p_{[a]}, \quad v_{[a] j}=\partial_{j} v_{[a]} .
$$


Substituting these expressions into Eqs. (33), (34), (36), (37) we obtain the expressions for the linearized Einstein's equations in $\Theta \mathrm{CDM}$,

$$
\begin{array}{r}
2 \Delta \psi-3 \mathcal{H}(2+\alpha B) \dot{\psi}-\alpha \Delta \phi-\frac{2\left(\bar{\rho}_{[\gamma]}+\bar{\rho}_{[\mathrm{cm}]}\right) a^{2}}{M_{0}^{2}} \phi+\alpha \Delta \dot{\chi}+\alpha(1-B) \mathcal{H} \Delta \chi \\
+\frac{\mu^{2} a}{M_{0}^{2}} \dot{\xi}-\frac{a^{2}\left(\delta \rho_{[\gamma]}+\delta \rho_{[c m]}\right)}{M_{0}^{2}}=0 \\
(2+\alpha B)(\dot{\psi}+\mathcal{H} \phi)+(\beta+\lambda) \Delta \chi-\frac{\left(\bar{\rho}_{[\gamma]}+\bar{p}_{[\gamma]}\right) a}{M_{0}^{2}} v_{[\gamma]}-\frac{\bar{\rho}_{[\mathrm{cm}]} a}{M_{0}^{2}} v_{[\mathrm{cm}]}=0 \\
3(2+\alpha B)\left[\ddot{\psi}+\mathcal{H}(\dot{\phi}+2 \dot{\psi})+\left(2 \dot{\mathcal{H}}+\mathcal{H}^{2}\right) \phi\right]+2 \Delta \phi-2 \Delta \psi \\
+\alpha B(\Delta \dot{\chi}+2 \mathcal{H} \Delta \chi)-\frac{3 a^{2} \delta p_{[\gamma]}}{M_{0}^{2}}=0 \\
\phi-\psi-\beta(\dot{\chi}+2 \mathcal{H} \chi)=0
\end{array}
$$

where $B$ and $\mathcal{H}$ are defined in (27), (28). These equations are respectively the (00), (0i), the trace and trace-free parts of the $(i j)$ Einstein's equations. In deriving (40c) we have used that the background metric satisfies the equation

$$
(2+\alpha B)\left(2 \dot{\mathcal{H}}+\mathcal{H}^{2}\right)-\frac{\mu^{4} c_{\Theta}^{2} a^{2}}{M_{0}^{2}}+\frac{2 \bar{p}_{[\gamma]} a^{2}}{M_{0}^{2}}=0
$$

which can be obtained by taking the time derivative of (10). Note that according to Eq. (40d) for non-zero $\beta$ the khronon field induces anisotropic stress and thus the gravitational potentials $\phi$ and $\psi$ are in general different.

The equations of motion (38) for the khronon and Goldstone field read at the linear order:

$$
\begin{gathered}
\ddot{\chi}+2 \mathcal{H} \dot{\chi}-c_{\chi}^{2} \Delta \chi+\left(\dot{\mathcal{H}}(1-B)+\mathcal{H}^{2}(1+B)+a^{2} c_{\Theta}^{4} k_{c}^{2}\right) \chi+\frac{a c_{\Theta}^{2} k_{c}^{2}}{\mu^{2}} \xi \\
=\dot{\phi}+\mathcal{H}(1+B) \phi+B \dot{\psi}, \\
\ddot{\xi}+2 \mathcal{H} \dot{\xi}-c_{\Theta}^{2} \Delta \xi-a \mu^{2} c_{\Theta}^{4} \Delta \chi=-a \mu^{2} c_{\Theta}^{2} \dot{\phi}-3 a \mu^{2} c_{\Theta}^{2} \mathcal{H} \phi,
\end{gathered}
$$

where we remind the definitions (18), (22) of $c_{\chi}$ and $k_{c}$. Note that these equations differ from Eqs. (29) valid in an external FRW universe by the appearance of the source term proportional to the metric perturbations on the r.h.s.

The perturbed matter equations coming from (39) have the standard form:

$$
\begin{aligned}
\delta \dot{\rho}_{[a]}+ & 3 \mathcal{H}\left(\delta \rho_{[a]}+\delta p_{[a]}\right)-\left(\bar{\rho}_{[a]}+\bar{p}_{[a]}\right)\left(\frac{\Delta v_{[a]}}{a}+3 \dot{\psi}\right)=0 \\
\dot{v}_{[a]}+3 \mathcal{H} v_{[a]}+\frac{\dot{\bar{\rho}}_{[a]}+\dot{\bar{p}}_{[a]}}{\bar{\rho}_{[a]}+\bar{p}_{[a]}} v_{[a]}-\frac{a \delta p_{[a]}}{\bar{\rho}_{[a]}+\bar{p}_{[a]}}-a \phi & =0 .
\end{aligned}
$$


In the next sections we analyze the system of linear equations (40), (41), (42).

\section{Cosmological perturbations: qualitative analysis}

We begin with a qualitative discussion of the evolution of cosmological perturbations in the $\Theta C D M$ model at radiation and matter domination. From Eqs. (40) it is easy to see that at these stages the modifications of the linearized equations of $\Theta C D M$, as compared to the $\Lambda \mathrm{CDM}$ case, are proportional to the dimensionless parameters $\alpha, \beta, \lambda$. As discussed in Sec. 2 , consistency with the experimental data requires these parameters to be small, $\alpha, \beta, \lambda \ll 1$. This allows to solve the system (40), (41), (42) with the following perturbative scheme. One first solves Eqs. (40), (42) in the zeroth-order approximation neglecting all the contributions proportional to $\alpha, \beta, \lambda$. Assuming adiabatic initial conditions, the solution is given by the standard adiabatic mode. For clarity we concentrate on the modes entering inside the horizon at the matter-dominated epoch. In this case the adiabatic mode is particularly simple: the two gravitational potentials $\phi$ and $\psi$ are equal and constant during radiation- and matterdominated epochs, with a jump at the radiation-matter equality,

$$
\psi^{(0)}=\phi^{(0)}= \begin{cases}\phi_{\gamma}=\text { const }, & \text { radiation domination; } \\ \phi_{c m}=\frac{9}{10} \phi_{\gamma}, & \text { matter domination }\end{cases}
$$

At the second step of the perturbative scheme these expressions are substituted into Eqs. (41) as the source for the fields $\chi$ and $\xi$. Finally, the solution for $\chi$ and $\xi$ is inserted back into Eqs. (40) to produce the corrections $\phi^{(1)}, \psi^{(1)}$ and $\left(\delta \rho_{[a]} / \rho_{[a]}\right)^{(1)}$ to the observable quantities. Thus our task at the moment is to solve Eqs. (41) with the sources (43).

A complete analysis should also include the evolution at the epoch of DE-domination. However, as this epoch started only recently it does not qualitatively affect the results. At the same time, all the following analytic estimates should be understood as giving only the qualitative picture. For example, they do not capture the transient behavior at the boundaries of the different dynamical regimes, which may be important due to finite duration of the corresponding epochs. We will neglect these effects in the rest of this section, and postpone their proper treatment to the numerical analysis (see Sec. 6). 


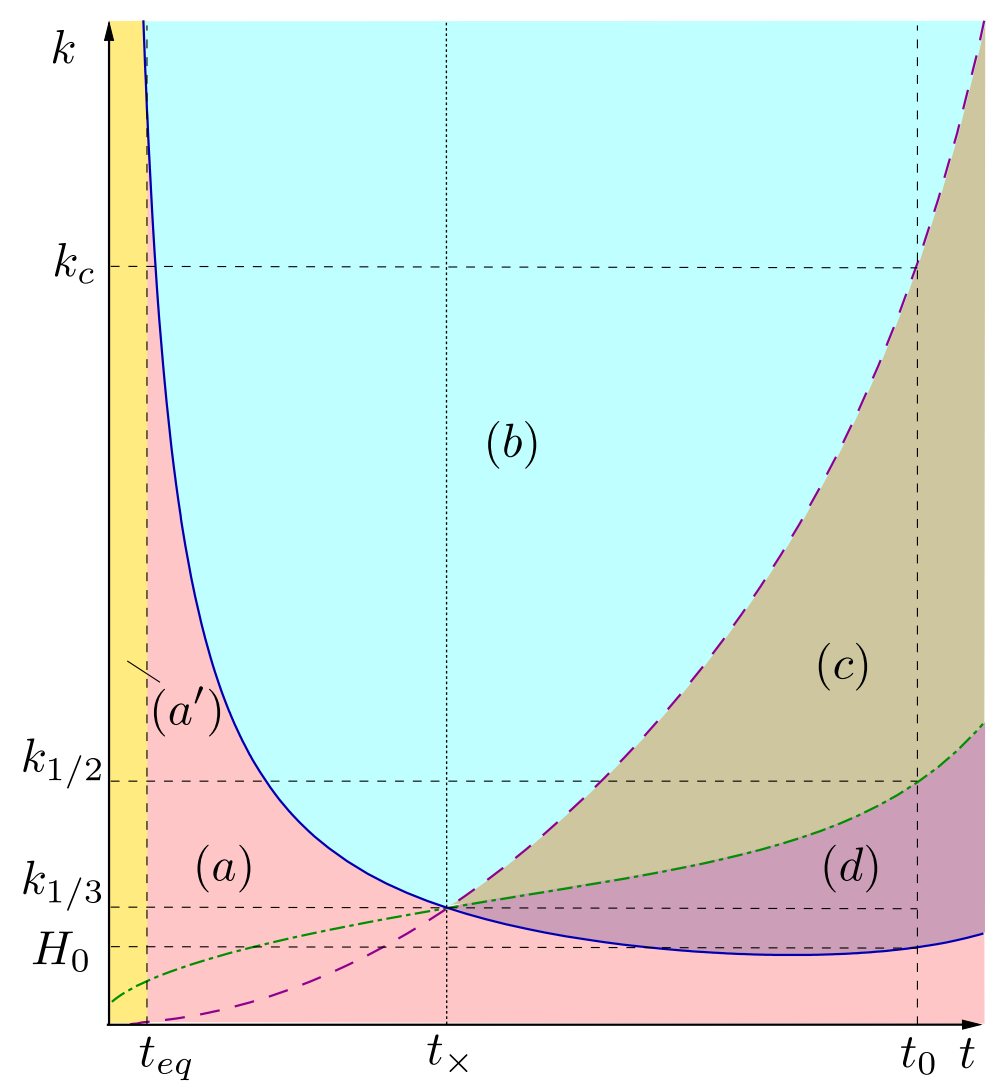

Figure 1: Time dependence of different physical scales relevant for the dynamics of cosmological perturbations: $\mathcal{H}$ (blue solid line), $a k_{c}$ (magenta dashed line), $\sqrt{\mathcal{H} a k_{c}}$ (green dash-dotted line). The time of the triple intersection of these curves is denoted by $t_{\times}$. The evolution of perturbations proceeds differently in the regions $\left(a^{\prime}\right),(a),(b),(c),(d)$. The time of radiationmatter equality is denoted by $t_{e q}$; $t_{0}$ is the present time and $H_{0}$ is the present Hubble rate. The present scale factor $a\left(t_{0}\right)$ is set to 1 . The beginning of current accelerated expansion roughly corresponds to the point where $\mathcal{H}$ starts growing. The definition of $k_{c}, k_{1 / 2}, k_{1 / 3}$ is given in the text.

\subsection{Different regimes of evolution}

One expects the behavior of a mode with a given comoving momentum $k$ to be different depending on the relation between $12 k$ and various scales that can be constructed out of the coefficients in Eqs. (41). Clearly, two important scales are the Hubble rate $\mathcal{H}$ and the

\footnotetext{
${ }^{12}$ In principle, the comparison of the different scales should include a dependence on the propagation velocities $c_{\Theta}$ and $c_{\chi}$. For simplicity we will consider the case where both quantities are close to one.
} 
comoving value of the critical momentum $a k_{c}$. Below we will encounter a third relevant scale, the geometric mean of the first two, $\sqrt{\mathcal{H} a k_{c}}$. The meaning of this latter scale can be understood as follows. For modes with $k=\sqrt{\mathcal{H} a k_{c}}$ the frequency (32) of the low-frequency branch is comparable to the expansion rate of the universe and therefore the modes with this and smaller $k$ are significantly affected by the Hubble friction. The dependence of the three scales on time is illustrated in Fig. 1. One distinguishes five different regimes of the mode evolution:

$\left(a^{\prime}\right)$ superhorizon modes, $k<\mathcal{H}$, radiation dominated universe;

(a) superhorizon modes, $k<\mathcal{H}$, matter dominated universe;

(b) subhorizon modes with $\mathcal{H}, a k_{c}<k$;

(c) subhorizon modes with $\mathcal{H}, \sqrt{\mathcal{H} a k_{c}}<k<a k_{c}$;

(d) subhorizon modes with $\mathcal{H}<k<\sqrt{\mathcal{H} a k_{c}}$.

For the following discussion it is convenient to normalize $k$ to be equal to the physical wave-number of the mode at present by setting the today scale factor to unity, $a\left(t_{0}\right)=1$. It is also useful to express $k_{c}$ in terms of the present Hubble rate $H_{0}$. The latter is given by the expression

$$
H_{0}=c_{\Theta} \mu^{2}\left(\frac{4 \pi G_{\text {cosm }}}{3 \Omega_{D E}}\right)^{1 / 2}
$$

where $\Omega_{D E} \approx 0.75$ is the dark energy density fraction. Recalling the definition (22) we obtain

$$
k_{c}=\frac{1}{c_{\Theta}}\left(\frac{6 \Omega_{D E}[1+\beta / 2+3 \lambda / 2]}{\alpha}\right)^{1 / 2} H_{0} .
$$

We see from Fig. 1 that depending on $k$ the different modes go through different regimes of evolution. Before studying these regimes case by case, let us briefly outline their broad features.

The modes with $k>k_{c}$ go only through the regimes $\left(a^{\prime}\right),(a)$ and $(b)$. According to the analysis of Sec. 3, in these regimes mixing between the $\chi$ - and $\xi$-perturbations is weak and both modes have linear dispersion relations with order one velocities. One expects that the effect of these modes on the growth of cosmological perturbations is at most of order $\alpha, \beta, \lambda$, as it is the case for pure Einstein-aether and khronon theories [35].

The dynamics of the modes with $k_{1 / 2}<k<k_{c}$ are more interesting. Here

$$
k_{1 / 2} \equiv \sqrt{H_{0} k_{c}}=\frac{1}{\sqrt{c_{\Theta}}}\left(\frac{6 \Omega_{D E}[1+\beta / 2+3 \lambda / 2]}{\alpha}\right)^{1 / 4} H_{0} .
$$

These modes spend some time in the regime $(c)$. We are going to see that in this range there are non-trivial effects due to $\chi-\xi$ mixing and the backreaction onto the gravitational 
potentials is enhanced by a factor $\alpha^{-1 / 2}$ compared to the short wave-length regime. Note that for $\alpha$ not extremely small, $\alpha>10^{-8}$, the momentum $k_{1 / 2}$ lies below the Hubble rate at the radiation-matter equality $13 \mathcal{H}_{e q} \sim 100 H_{0}$. Thus the modes with $k \sim k_{1 / 2}$ enter the horizon at the matter-dominated epoch.

The modes with $k_{1 / 3}<k<k_{1 / 2}$ pass through all five regimes $\left(a^{\prime}\right)-(d)$. The momentum $k_{1 / 3}$ corresponds to the intersection of the lines $\mathcal{H}(t)$ and $a(t) k_{c}$, see Fig. 11. To determine it we note that for the interesting values of the parameters the intersection happens at the epoch of matter domination. During this epoch the scale factor is given by

$$
a=A_{c m} t^{2}, \quad A_{c m} \equiv \frac{\Omega_{c m} H_{0}^{2}}{4}
$$

where $\Omega_{c m} \approx 0.25$ is the matter density fraction. This gives for the time of the intersection

$$
t_{\times}=\left(\frac{8}{\Omega_{c m} H_{0}^{2} k_{c}}\right)^{1 / 3}
$$

and hence,

$$
k_{1 / 3}=\mathcal{H}\left(t_{\times}\right)=\left(\Omega_{c m} H_{0}^{2} k_{c}\right)^{1 / 3}=\left(\frac{\Omega_{c m}}{c_{\Theta}}\right)^{1 / 3}\left(\frac{6 \Omega_{D E}[1+\beta / 2+3 \lambda / 2]}{\alpha}\right)^{1 / 6} H_{0} .
$$

For these modes we will also find a relative enhancement of the backreaction onto the gravitational potentials of order $\alpha^{-1 / 2}$.

Finally, the modes with $k<k_{1 / 3}$ go directly from the regime $(a)$ to the regime $(d)$ and do not have enough time to develop. Thus the backreaction of these modes on the gravitational potentials is expected to be $O(\alpha)$. Note that for these modes to be observable, their wave-length must be inside the present horizon size, $k>H_{0}$.

We now confirm the statements made above by the detailed analysis of the listed regimes.

\section{Regime $\left(a^{\prime}\right): k<\mathcal{H}$ during radiation domination.}

As we are considering superhorizon modes, we can neglect all terms with spatial Laplacians in Eqs. (41). Some care is needed with the last term on the l.h.s. of (41b) as it is the only term in this equation containing the field $\chi$; we will check explicitly below that this term is indeed small on the solutions.

\footnotetext{
${ }^{13}$ This relation is obtained as follows. At the matter-dominated epoch $a \propto t^{2}$ and $\mathcal{H}=2 / t$. Hence, $\mathcal{H}_{e q}=H_{0}\left(t_{0} / t_{e q}\right)=H_{0} \sqrt{z_{e q}+1}$, where $z_{e q} \sim 10^{4}$ is the corresponding red-shift.
} 
At the radiation-dominated stage the scale factor depends linearly on time,

$$
a=A_{\gamma} t, \quad A_{\gamma} \equiv \sqrt{\Omega_{\gamma}} H_{0},
$$

where $\Omega_{\gamma} \sim 10^{-5}$ is the radiation density fraction today. Substituting this and (43) into Eqs. (41) we obtain

$$
\begin{aligned}
& \ddot{\chi}+\frac{2}{t} \dot{\chi}+\left[\frac{2 B}{t^{2}}+A_{\gamma}^{2} t^{2} c_{\Theta}^{4} k_{c}^{2}\right] \chi+\frac{A_{\gamma} t c_{\Theta}^{2} k_{c}^{2}}{\mu^{2}} \xi=\frac{1+B}{t} \phi_{\gamma}, \\
& \ddot{\xi}+\frac{2}{t} \dot{\xi}=-3 A_{\gamma} \mu^{2} c_{\Theta}^{2} \phi_{\gamma} .
\end{aligned}
$$

This system has the solution:

$$
\chi=\frac{\phi_{\gamma} t}{2}, \quad \xi=-\frac{A_{\gamma} \mu^{2} c_{\Theta}^{2} \phi_{\gamma} t^{2}}{2} .
$$

It is straightforward to check that on this solution the ratio of the term $\Delta \chi$ in (41b) to the term $\ddot{\xi}$ is of order $c_{\Theta}^{2} k^{2} t^{2}$ which is indeed small for superhorizon modes.

Note that despite the apparent growth of the fields $\chi, \xi$ in (50), the physical perturbations remain subdominant with respect to the background. Indeed, combining (50) with the expressions (16), (25) for the background we obtain the total values of the fields $\varphi$ and $\Theta$ :

$$
\begin{aligned}
& \varphi=t\left(1+\phi_{\gamma} / 2\right), \\
& \Theta=-\frac{\mu^{2} c_{\Theta}^{2} A_{\gamma} t^{2}}{2}\left(1+\phi_{\gamma}\right) .
\end{aligned}
$$

We observe that the relative corrections are small. The solution (50) is equivalent to the time shift

$$
t \mapsto t\left(1+\phi_{\gamma} / 2\right)
$$

that corresponds precisely to the adiabatic mode at radiation domination.

Let us check whether the solution (50) is an attractor. To this end consider the solutions of the corresponding homogeneous equations (i.e. Eqs. (49) with zero r.h.s.). Those are particularly simple under the assumption that the second term in the square brackets in (49a) can be neglected. This is equivalent to the assumption

$$
\mathcal{H} \gg a k_{c},
$$

which is always satisfied during radiation domination for reasonable choices of parameters. Then the solution of the homogeneous equations is

$$
\begin{aligned}
\chi_{h o m} & =-\frac{A_{\gamma} c_{\Theta}^{2} k_{c}^{2}}{2(6+B) \mu^{2}} \xi_{0} t^{3}-\frac{A_{\gamma} c_{\Theta}^{2} k_{c}^{2}}{2(3+B) \mu^{2}} C t^{2}+D_{+} t^{q_{+}}+D_{-} t^{q_{-}}, \\
\xi_{\text {hom }} & =\xi_{0}+\frac{C}{t}
\end{aligned}
$$


where

$$
q_{ \pm}=\frac{-1 \pm \sqrt{1-8 B}}{2}
$$

and $\xi_{0}, C, D_{+}, D_{-}$are integration constants.

Let us first discuss the contributions proportional to $\xi_{0}$ and $C$. As long as we concentrate on the field $\xi$, the role of these contributions decreases with time compared to the adiabatic mode (50). Moreover, the constant mode $\xi_{0}$ is irrelevant when we are interested in the backreaction of $\xi$ on the gravitational potentials. Indeed, the only place where $\xi$ appears in the Einstein's equations is Eq. (40a) and there it enters with a time derivative. On the other hand, the terms proportional to $\xi_{0}$ and $C$ in the homogeneous solution (52a) for $\chi$ grow with time faster than the adiabatic mode and may become important at late times. Whether this happens or not depends on the initial conditions for $\xi$ which set the values of $\xi_{0}$ and $C$. A proper determination of these initial conditions would require analysis of the evolution of the system at the epoch prior to radiation-domination (i.e. at inflation and reheating) and also a precise model for the origin of the field $\Theta$ (e.g. if it exists during all the history of the universe, or appears as the result of a phase transition at some particular epoch). These issues are outside the scope of the present article. Nevertheless, let us give an argument showing that $\xi_{0^{-}}$and $C$-contributions into $\chi$ are likely to be small. For concreteness we concentrate on the $\xi_{0}$-contribution. Consider the ratio of this contribution to the adiabatic mode (50). Omitting factors of order one and using (14), (48) it can be written in the form,

$$
A_{\gamma} t^{2} k_{c} \cdot \frac{\xi_{0}}{\phi_{\gamma} M_{\alpha}} \sim \frac{a k_{c}}{\mathcal{H}} \cdot \frac{\sqrt{\epsilon} \xi_{0}}{\sqrt{\alpha} H_{i n f}}
$$

where $H_{\text {inf }}$ is the Hubble rate at inflation and $\epsilon$ is the inflationary slow-roll parameter. In passing to the second expression we have used the standard formula

$$
\phi_{\gamma} \sim \sqrt{\frac{G_{\operatorname{cosm}}}{\epsilon}} H_{\text {inf }},
$$

for the amplitude of the primordial perturbations in the potentially driven slow-roll inflation. The first factor in (53) is small due to the inequality (51). Let us estimate the second factor. Assuming that the fluctuations of the $\xi$-field are generated at the inflationary epoch, we have the estimate $\xi_{0} \lesssim H_{\text {inf }}$. We will be interested in the values of $\alpha$ in the range from $10^{-4}$ to $10^{-2}$. Finally, recalling that the measurements of the tilt of the primordial spectrum give an upper bound $\epsilon \lesssim 10^{-2}$, we obtain that the second factor in (53) can be at most $\lesssim 10$. Overall, we conclude that the product (53) is small in general. A similar reasoning applies 
to the contribution in (52a) that is proportional to $C$. We will neglect these contributions in what follows.

Consider now the last two terms in (52a). The term proportional to $D_{-}$always decays with time and can be safely neglected. However, the $D_{+}$term can either decay or grow depending on the sign of the parameter $B$. Moreover, for $B<-1$ this term grows faster than the adiabatic contribution (50). We have already encountered this growing regime in Sec. 3, where it was related to the possibility of having negative khronon mass squared. Below we will only consider the case $B>-1$ where the leading solution is given by the adiabatic mode (150).

\section{Regime $(a): k<\mathcal{H}$ during matter domination.}

During matter domination the scale factor is given by the formula (46). Substituting this into Eqs. (41) and neglecting the terms with spatial Laplacians we obtain

$$
\begin{aligned}
& \ddot{\chi}+\frac{4}{t} \dot{\chi}+\left[\frac{2(1+3 B)}{t^{2}}+A_{c m}^{2} t^{4} c_{\Theta}^{4} k_{c}^{2}\right] \chi+\frac{A_{c m} t^{2} c_{\Theta}^{2} k_{c}^{2}}{\mu^{2}} \xi=\frac{2(1+B)}{t} \phi_{c m}, \\
& \ddot{\xi}+\frac{4}{t} \dot{\xi}=-6 A_{c m} t \mu^{2} c_{\Theta}^{2} \phi_{c m} .
\end{aligned}
$$

The adiabatic mode is also a solution and has the form,

$$
\chi=\frac{\phi_{c m} t}{3}, \quad \xi=-\frac{A_{c m} \mu^{2} c_{\Theta}^{2} \phi_{c m} t^{3}}{3} .
$$

It corresponds to the time shift

$$
t \mapsto t\left(1+\phi_{c m} / 3\right)
$$

Similarly to the previous case one can check that the term proportional to $\Delta \chi$ on (41b) can be safely ignored. Also, as in the previous case, one can argue that the solutions of the homogeneous equations are irrelevant, apart from one, possibly growing, mode. This mode appears in the regime (51) and has the form

$$
\chi_{\text {hom }} \propto t^{r_{+}}, \quad r_{+}=\frac{-3+\sqrt{1-24 B}}{2} .
$$

It grows faster than the adiabatic mode if $B<-1$. This growth is cut off once the mode enters inside the horizon or the inequality (51) is violated. Below we restrict to the stable case $B>-1$. 


\section{Regime (b): subhorizon modes with $a k_{c}<k$.}

We now turn to the evolution of the subhorizon perturbations. We shall concentrate on the modes that enter inside the horizon at the matter-dominated stage. Neglecting the $\mathcal{H}$ dependent terms in brackets in Eq. (41a) and substituting (43) on the r.h.s. we obtain,

$$
\begin{aligned}
& \ddot{\chi}+\frac{4}{t} \dot{\chi}+\left[c_{\chi}^{2} k^{2}+A_{c m}^{2} t^{4} c_{\Theta}^{4} k_{c}^{2}\right] \chi+\frac{A_{c m} t^{2} c_{\Theta}^{2} k_{c}^{2}}{\mu^{2}} \xi=\frac{2(1+B)}{t} \phi_{c m}, \\
& \ddot{\xi}+\frac{4}{t} \dot{\xi}+c_{\Theta}^{2} k^{2} \xi+A_{c m} t^{2} \mu^{2} c_{\Theta}^{4} k^{2} \chi=-6 A_{c m} t \mu^{2} c_{\Theta}^{2} \phi_{c m} .
\end{aligned}
$$

Deep inside the regime under study, $k \gg a k_{c}$, the previous equations have the approximate solution:

$$
\chi=\frac{2(1+B) \phi_{c m}}{c_{\chi}^{2} k^{2} t}, \quad \xi=-2\left(3+\frac{c_{\Theta}^{2}}{c_{\chi}^{2}}(1+B)\right) \frac{A_{c m} \mu^{2} \phi_{c m} t}{k^{2}} .
$$

This solution decays with time (in the case of the $\xi$-perturbation it must be compared with the behavior $\bar{\Theta} \propto t^{3}$ of the background). On top of (57) the fields $\chi$, $\xi$ exhibit oscillations with frequencies $\omega_{\chi}, \omega_{\xi}$ (Eqs. (23)) and amplitudes decaying as $1 / t^{2}$. These oscillations represent small corrections to the approximate solution (57) which disappear at large times.

Regime $(c)$ : subhorizon modes with $\sqrt{\mathcal{H} a k_{c}}<k<a k_{c}$.

According to the results of Sec. 3, in this regime one of the branches of $\chi-\xi$ perturbations has a frequency gap of order $k_{c}$, see Eq. (31). As long as we consider frequencies smaller than the gap, this mode can be integrated out. To obtain the equations for the remaining

mode we use the following trick. First, we eliminate the explicit dependence of the equations on the parameter $\mu$ by introducing

$$
\tilde{\xi}=\xi / \mu^{2} .
$$

The second step is to take the limit

$$
k_{c} \rightarrow \infty
$$

The physical meaning of this limit is that we focus on the modes with frequencies $\omega$ much smaller than the gap (cf. (32)). At the same time we must be careful to keep the frequency of these modes non-zero. From Eq. (32) we read out $\omega_{-} \propto k^{2} / k_{c}$. Thus to keep $\omega_{-}$fixed (58a) must be accompanied by

$$
k \rightarrow \infty, \quad k \propto \sqrt{k_{c}} .
$$


The rest of the quantities in the equations must remain finite14. In this way Eq. (56a) reduces to

$$
\left[\frac{c_{\chi}^{2} k^{2}}{A_{c m} t^{2} c_{\Theta}^{2} k_{c}^{2}}+A_{c m} t^{2} c_{\Theta}^{2}\right] \chi+\tilde{\xi}=0
$$

where we have kept the main subleading contribution represented by the first term in the brackets. Substituting this into (56b) we obtain,

$$
\ddot{\tilde{\xi}}+\frac{4}{t} \dot{\tilde{\xi}}+\frac{c_{\chi}^{2} k^{4}}{A_{c m}^{2} t^{4} c_{\Theta}^{2} k_{c}^{2}} \tilde{\xi}=-6 A_{c m} t c_{\Theta}^{2} \phi_{c m} .
$$

In the regime $(c)$ that we are considering now, the leading solution is obtained by neglecting the terms with time derivatives on the l.h.s. of (59b). This gives,

$$
\chi=\frac{6 A_{c m}^{2} c_{\Theta}^{2} k_{c}^{2} t^{3} \phi_{c m}}{c_{\chi}^{2} k^{4}}, \quad \xi=-\frac{6 A_{c m}^{3} \mu^{2} c_{\Theta}^{4} k_{c}^{2} t^{5} \phi_{c m}}{c_{\chi}^{2} k^{4}},
$$

where the expression for $\chi$ is obtained using (59a). Clearly, these perturbations grow with time. As anticipated at the end of Sec. 3 this growth results from the low propagation speed of these modes.

On top of the previous solution there is an oscillatory mode that corresponds to the solution of the homogeneous part of $(59 \mathrm{~b})$,

$$
\xi_{\text {hom }} \propto \frac{1}{t} \cdot \exp \left( \pm i \frac{c_{\chi} k^{2}}{A_{c m} c_{\Theta} k_{c} t}\right)
$$

However, it rapidly decays compared to (60) and hence is irrelevant.

Regime $(d)$ : subhorizon modes with $k<\sqrt{\mathcal{H} a k_{c}}$.

In this case the frequency of the modes is lower than the Hubble rate and we can neglect the third term on the 1.h.s. of (59b). Then the perturbations are frozen and we obtain the same adiabatic solution (55) as in the superhorizon case. It is important to stress though, that the modes we are discussing now have wave-lengths shorter than the Hubble size and are therefore observable.

\subsection{Corrections to the metric perturbations}

According to the iterative procedure described at the beginning of the current section, we shall substitute the expressions for the $\chi$ and $\xi$ perturbations obtained above into the Einstein's Eqs. (40). This will give us the corrections to the observable quantities due to the

\footnotetext{
${ }^{14}$ An alternative derivation of this limit and Eqs. (59) involves diagonalization of the quadratic action (26).
} 
presence of these fields. It is convenient to concentrate on Eq. (40c). Substituting $\phi$ in terms of $\psi$ and $\chi$ from (40d) we obtain

$$
\begin{aligned}
\ddot{\psi} & +3 \mathcal{H} \dot{\psi}+\left(2 \dot{\mathcal{H}}+\mathcal{H}^{2}\right) \psi-\frac{a^{2} \delta p_{[\gamma]}}{(2+\alpha B) M_{0}^{2}}= \\
& -\frac{\beta+\lambda}{2+\alpha B}(\Delta \dot{\chi}+2 \mathcal{H} \Delta \chi)-\beta\left[\mathcal{H} \ddot{\chi}+\left(2 \dot{\mathcal{H}}+3 \mathcal{H}^{2}\right) \dot{\chi}+\left(6 \dot{\mathcal{H}} \mathcal{H}+2 \mathcal{H}^{3}\right) \chi\right]
\end{aligned}
$$

The r.h.s. represents the source for the correction $\psi^{(1)}$ to the standard adiabatic behavior (43) of $\psi$. Let us compare the two terms on the r.h.s. For superhorizon modes the second term dominates. Clearly, it gives a contribution of order $O(\beta)$ to $\psi^{(1)}$. On the other hand, for subhorizon modes the first term becomes dominant. Indeed, it is enhanced compared to the second term by the ratio $(k / \mathcal{H})^{2}$. Below we focus on the contribution of this term into $\psi^{(1)}$ and neglect the second term on the r.h.s. of (61).

Assuming as before that the relevant modes enter the horizon at the matter-dominated epoch, Eq. (61) is further simplified to

$$
\ddot{\psi}+\frac{6}{t} \dot{\psi}=\frac{(\beta+\lambda) k^{2}}{2}\left(\dot{\chi}+\frac{4}{t} \chi\right)
$$

This yields for $\psi^{(1)}$ the expression

$$
\psi^{(1)}(t)=\frac{(\beta+\lambda) k^{2}}{2} \int_{t_{*}}^{t} \frac{\mathrm{d} t^{\prime}}{t^{\prime 6}} \int_{t_{*}}^{t^{\prime}} \mathrm{d} t^{\prime \prime} t^{\prime \prime 6}\left(\dot{\chi}\left(t^{\prime \prime}\right)+\frac{4}{t^{\prime \prime}} \chi\left(t^{\prime \prime}\right)\right),
$$

where $t_{*}$ is the time of horizon crossing for the mode with given $k$. At this time, the adiabatic initial conditions imply $\psi^{(1)}\left(t_{*}\right) \approx 0$.

Consider first the modes with $k_{c}<k$. As it is clear from Fig. 1, these modes spend all subhorizon evolution in the regime $(b)$, where the $\chi$-field is given by (57). Inserting this into (62) gives,

$$
\psi^{(1)} \sim(\beta+\lambda) \cdot \frac{3(1+B)}{5 c_{\chi}^{2}} \phi_{c m} \ln \frac{t}{t_{*}} .
$$

We see that for these short modes the correction to the gravitational potential remains parametrically small, i.e. $O(\beta+\lambda)$. Notice, however, that the numerical value of the logarithmic factor in (63) can be quite large (of order 10), which can lead to a sizable effect if $\beta, \lambda$ are not too small.

Next we turn to the modes with $k_{1 / 2}<k<k_{c}$, see Fig. 1. Due to fast growth of these modes in the region $(c)$, the integrals in (62) are saturated at the upper end of the integration 
domain. Using (60) we get an estimate for the value of $\psi^{(1)}$ at present 15 :

$$
\psi^{(1)}\left(t_{0}\right) \approx \frac{7}{12}(\beta+\lambda) \frac{c_{\Theta}^{2} k_{c}^{2}}{c_{\chi}^{2} k^{2}} \phi_{c m} .
$$

At $k \approx k_{1 / 2}$ we obtain

$$
\left.\psi^{(1)}\left(t_{0}\right)\right|_{k=k_{1 / 2}} \approx \frac{7 \sqrt{6 \Omega_{D E}} c_{\Theta}}{12 c_{\chi}^{2}} \cdot \frac{\beta+\lambda}{\sqrt{\alpha}} \phi_{c m},
$$

where we have used Eqs. (44), (45). We clearly see the parametric enhancement of the signal to the leve16 $O(\sqrt{\alpha})$.

Finally, we consider the modes with $H_{0}<k<k_{1 / 2}$. Again, the integral (62) is saturated at the upper end corresponding to the regime $(d)$. Using the appropriate formula for $\chi$, Eq. (55), we obtain

$$
\psi^{(1)}\left(t_{0}\right) \approx \frac{5}{84}(\beta+\lambda)\left(k t_{0}\right)^{2} \phi_{c m} \approx \frac{5}{21}(\beta+\lambda)\left(\frac{k}{H_{0}}\right)^{2} \phi_{c m},
$$

where in passing to the last expression we have used $H_{0} \approx 2 / t_{0}$. This expression has a maximum at the highest momenta of the interval, $k \sim k_{1 / 2}$. Using (45) one readily verifies that, up to a numerical factor of order one, this expression matches at $k \sim k_{1 / 2}$ with the estimate (64).

To sum up, the analytical study of this section shows that the correction $\psi_{k}^{(1)}$ to the present-day amplitude of the gravitational potential has a maximum of order $\sqrt{\alpha} \phi_{c m}$ at $k \sim k_{1 / 2}$ with the falloff $\psi^{(1)} \propto k^{2}, \psi^{(1)} \propto k^{-2}$ on the two sides, and a logarithmic tale $\psi^{(1)} \sim(\beta+\lambda) \phi_{c m} \ln k$ extending towards large momenta $k>k_{c}$. This will be verified by the numerical results in the next section.

\section{Cosmological perturbations: numerical results}

In this section we report the results of numerical integration of Eqs. (40), (41), (42). We consider the density fractions of radiation and cold matter today

$$
\Omega_{\gamma}=5 \cdot 10^{-5}, \quad \Omega_{c m}=0.25 .
$$

\footnotetext{
${ }^{15}$ Strictly speaking, the formula (62) is applicable only during matter-domination. However, for the reasons discussed at the beginning of this section, we neglect possible corrections due to the start of the accelerated cosmological expansion.

${ }^{16}$ Recall that we assume the parameters $\alpha, \beta, \lambda$ to be of the same order.
} 

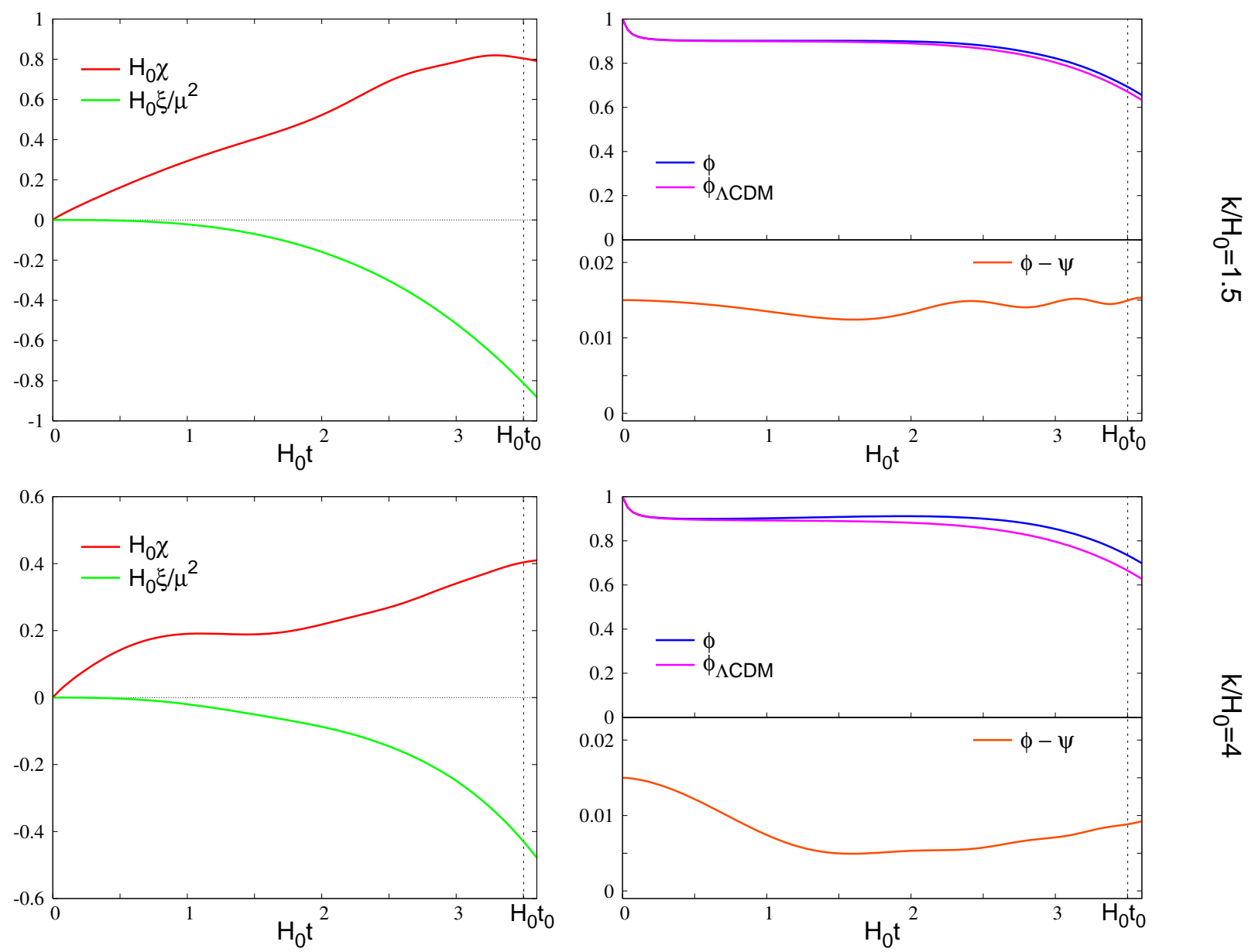

Figure 2: Solution of the system (40), (41), (42) for two values of the momentum: $k=1.5 H_{0}$ (upper panels), $k=4 H_{0}$ (lower panels), where $H_{0}$ is the present Hubble constant. Left panels show the dependence of the dark energy perturbations $\chi, \xi$ on conformal time $t$. Right panels show the perturbations of the gravitational potential $\phi$ and the difference $(\phi-\psi)$ between the two gravitational potentials. For comparison we also present the dependence of the gravitational potential in the standard $\Lambda$ CDM cosmology. The parameters of the model are $\alpha=0.02, \beta=0.01, \lambda=0.01$. For these values $k_{c}=15.15 H_{0}, k_{1 / 2}=3.89 H_{0}, k_{1 / 3}=1.56 H_{0}$. Present time corresponds to $t_{0}=3.5 H_{0}^{-1}$.

Correspondingly, the dark energy fraction is

$$
\Omega_{D E}=0.75
$$



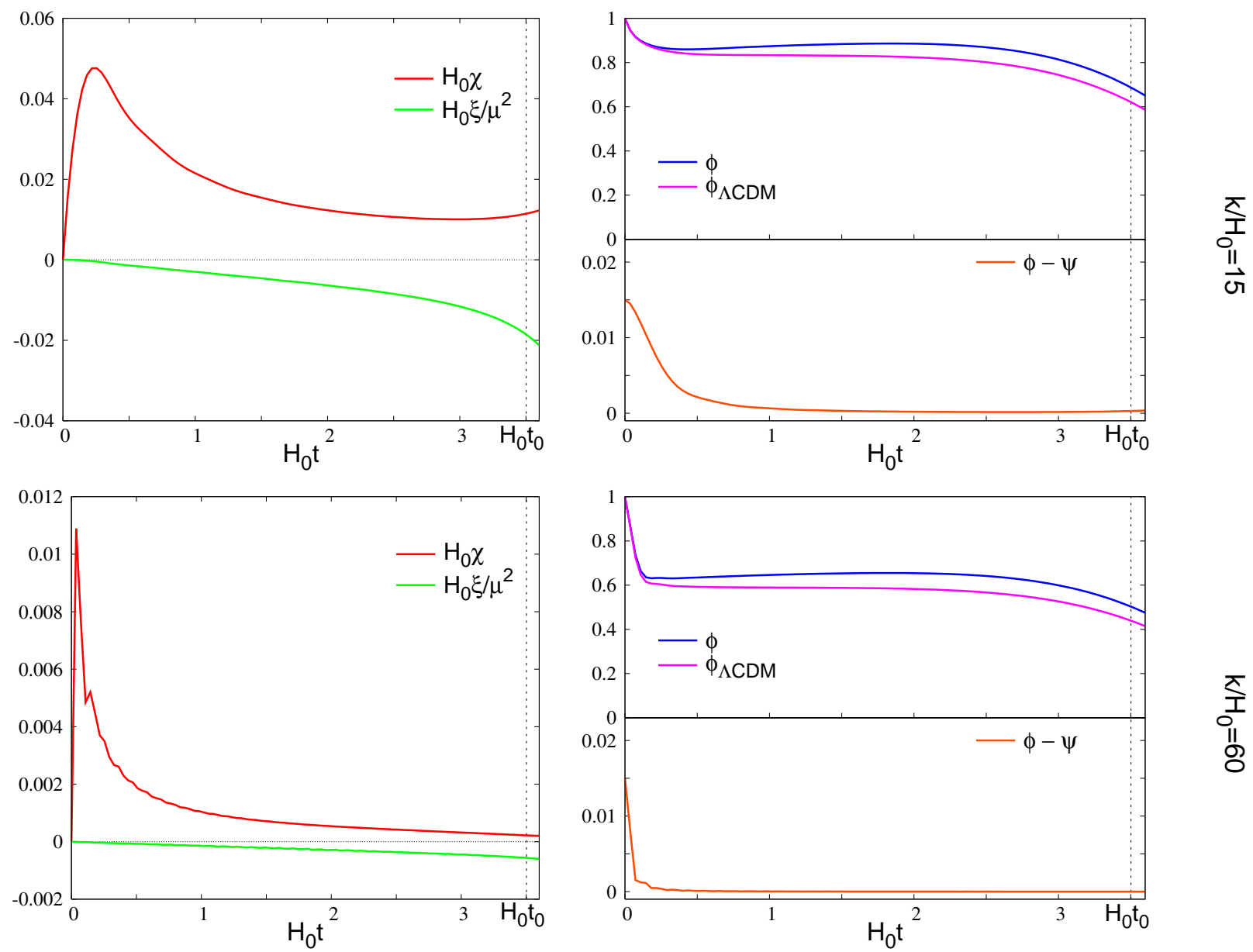

Figure 3: The same as Fig. 2 for $k=15 H_{0}$ (upper panels), $k=60 H_{0}$ (lower panels).

Let us emphasize again that for simplicity we neglect all effects related to baryon-photon coupling and merely include baryons into the cold matter fraction. Similarly, we neglect the neutrino masses. Still, within these simplifications we refer to the cosmological model with $\mathrm{CC}$ and without $\xi$ and $\chi$ perturbations as $\Lambda \mathrm{CDM}$. Details of the numerical procedure are presented in the Appendix A. Here we summarize the results.

The time dependence of the fields $\chi, \xi$, the gravitational potential $\phi$ and the difference $(\phi-\psi)$ is shown in Figs. 2 and 3 for several values of the momentum $k$. The parameters of the model are taken to be

$$
\alpha=0.02, \quad \beta=0.01, \quad \lambda=0.01, \quad c_{\Theta}=1 .
$$




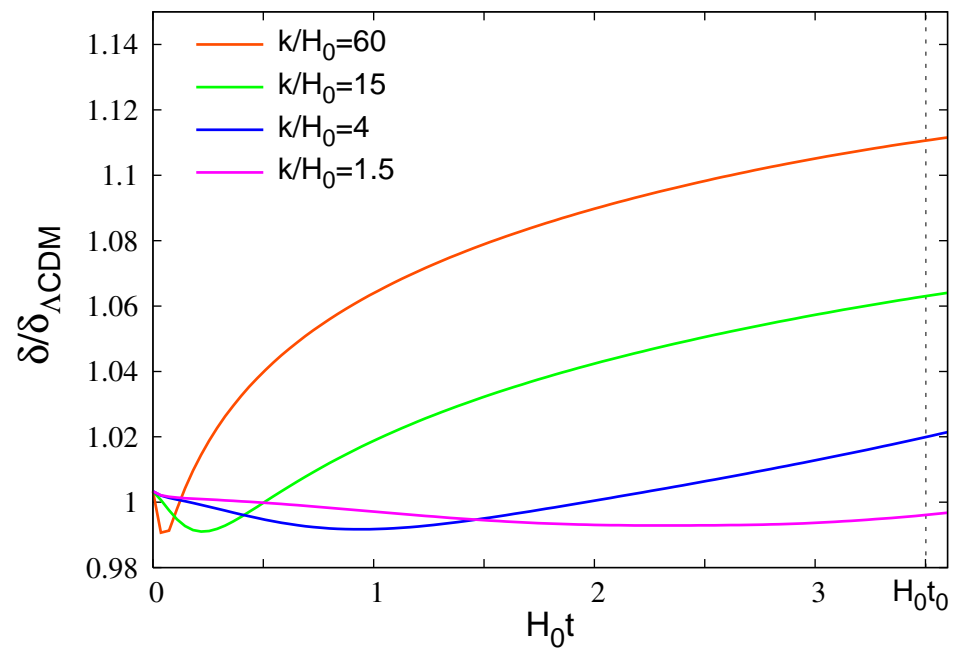

Figure 4: The ratio of density contrasts in the $\Theta C D M$ and $\Lambda$ CDM models versus time for several values of the mode momentum $k$. The values of parameters are the same as for Fig. 2,

This choice satisfies the PPN17, BBN and gravitational radiation constraints [17, 28]. The initial conditions correspond to the adiabatic mode, and, for illustration purposes, the initial value of $\phi$ is normalized to 1 for every momentum. We also show for comparison the dependence of the Newton potential on time for the standard $\Lambda$ CDM case (where $\psi=\phi$ within our approximation).

We see that perturbations of the gravitational potential are enhanced at late times compared to the $\Lambda$ CDM case. The relative magnitude of enhancement is of order $10 \%$ which agrees with the analytic estimate $\sqrt{\alpha} \approx 0.14$ derived in the previous section. On the other hand, the difference between the two gravitational potentials $(\phi-\psi)$ which is initially of order $10^{-2}$ rapidly decreases once the mode enters into the horizon, and as a consequence is at present negligible for $k$ larger than $k_{1 / 2}$. Note that the overall amplitude $10^{-2}$ for long wavelength modes agrees with the estimates $(\phi-\psi) \sim \alpha=0.02$.

One expects that the enhancement in the gravitational perturbations will lead to the increase of structure growth rate. To illustrate this point we plot in Fig. 4 the ratio $\delta / \delta_{\Lambda C D M}$, where $\delta$ and $\delta_{\Lambda C D M}$ are the cold matter density contrasts in the case of the present model

\footnotetext{
${ }^{17}$ Recall that $\alpha=2 \beta$ case avoids the PPN bounds.
} 


\begin{tabular}{|c|c|c|c|c|c|}
\hline & $\alpha$ & $\beta$ & $\lambda$ & $k_{c}\left(\mathrm{~h} \mathrm{Mpc}^{-1}\right)$ & $k_{1 / 2}\left(\mathrm{~h} \mathrm{Mpc}^{-1}\right)$ \\
\hline $\mathrm{a}$ & $2 \cdot 10^{-2}$ & $10^{-2}$ & $10^{-2}$ & $5.1 \cdot 10^{-3}$ & $1.3 \cdot 10^{-3}$ \\
\hline $\mathrm{b}$ & $2 \cdot 10^{-3}$ & $10^{-3}$ & $10^{-3}$ & $1.6 \cdot 10^{-2}$ & $2.3 \cdot 10^{-3}$ \\
\hline $\mathrm{c}$ & $2 \cdot 10^{-4}$ & $10^{-4}$ & $10^{-4}$ & $5.0 \cdot 10^{-2}$ & $4.1 \cdot 10^{-3}$ \\
\hline $\mathrm{d}$ & $10^{-4}$ & 0 & $10^{-4}$ & $7.1 \cdot 10^{-2}$ & $4.9 \cdot 10^{-3}$ \\
\hline
\end{tabular}

Table 1: Model parameters corresponding to the curves in Fig. [5]and Fig. 6] and the respective values of momenta $k_{c}, k_{1 / 2}$.

and $\Lambda \mathrm{CDM}$ respectively,

$$
\delta \equiv \frac{\delta \rho_{[c m]}}{\bar{\rho}_{[c m]}} .
$$

As expected we observe the increase in the growth of structure at recent times. The relative effect is stronger for shorter modes and can be as large as $11 \%$ for our choice of parameters.

The next plots show the comparison between the power spectra of perturbations in the $\Theta \mathrm{CDM}$ and $\Lambda \mathrm{CDM}$ cosmologies evaluated at the present moment of time. Fig. [5] shows the relative differences of the power spectra for the gravitational potential $\phi$ and the cold matter density contrast $\delta$,

$$
\Delta_{\phi}(k) \equiv \frac{P_{\phi}(k)}{P_{\phi_{\Lambda C D M}}(k)}-1, \quad \Delta_{\delta}(k) \equiv \frac{P_{\delta}(k)}{P_{\delta_{\Lambda C D M}}(k)}-1 .
$$

These differences were computed for several values of the model parameters that are listed in Table 1. We also present the corresponding values of the momenta $k_{c}$ and $k_{1 / 2}$. Note that, as expected from the results of the previous section, the functions $\Delta_{i}(k)$ peak around $k \approx k_{1 / 2}$ (different for the different sets of parameters) with a tail extending to larger momenta. In agreement with the analytical results, the value of the functions $\Delta_{i}$ at the peak scales approximately as $\sqrt{\alpha}$ while the tails are proportional to $\alpha$.

Finally, we plot in Fig. [6 the spectrum of the relative difference $(\phi-\psi) / \phi$ between the two gravitational potentials in $\Theta \mathrm{CDM}$. This difference is completely negligible at $k$ larger than $0.01 \mathrm{Mpc}^{-1}$. At smaller $k$ it can be as large as a few percent for certain choices of parameters.

We stress once again that all above results are valid in the case of longitudinal perturbations in the Einstein-aether model coupled to the $\Theta$-field. $\chi$ in this case corresponds to the longitudinal component of the aether perturbations. 

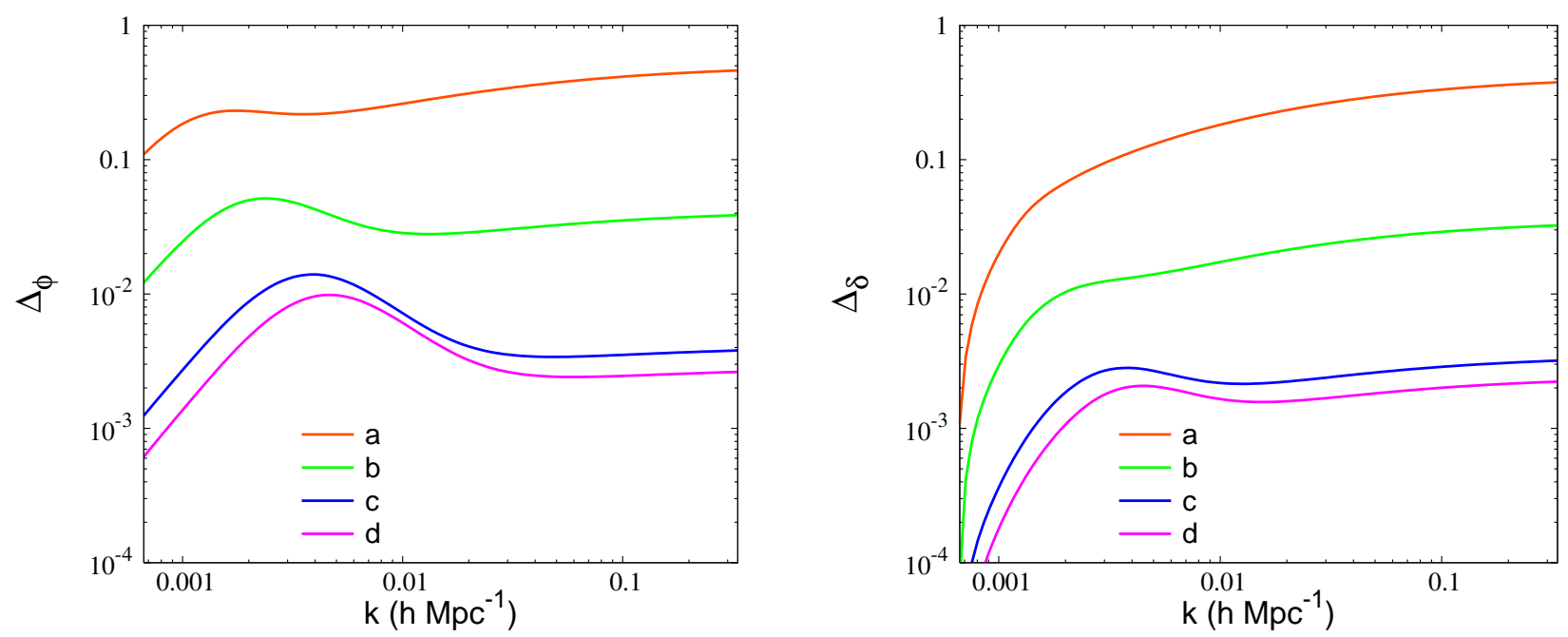

Figure 5: Comparison between the power spectra for the gravitational potential (left panel) and the matter density contrast (right panel) in the $\Theta C D M$ and $\Lambda$ CDM cosmologies. The curves correspond to the values of the model parameters listed in Table 1 .

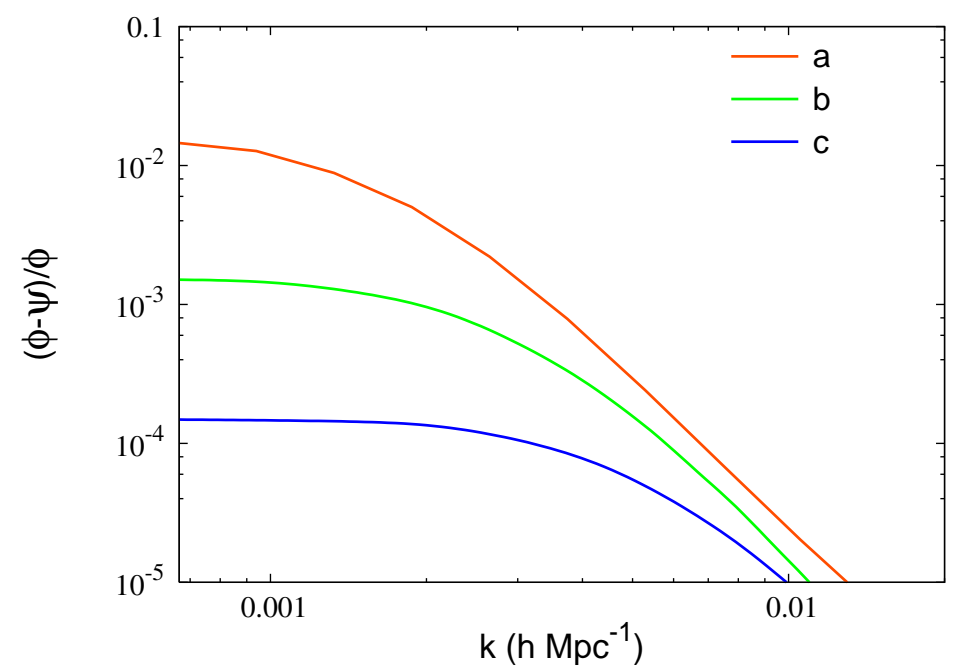

Figure 6: The difference between two scalar gravitational potentials for several choices of parameters listed in Table 1 .

\section{Conclusions}

In this paper we have proposed a dark energy model based on the idea that Lorentz invariance is not an exact symmetry of nature and is broken in the gravitational sector. As the 
framework for Lorentz breaking we considered the theory of a unit time-like vector field aether. This can be either a general unit vector, in which case the setup corresponds to the Einstein-aether model, or it can be expressed in terms of the gradient of a scalar field - khronon - that defines a global time coordinate. Dark energy appears in this framework in a rather generic and simple way due to an additional massless scalar with an exact shift symmetry and arbitrary relevant couplings to the aether compatible with this invariance. An example of such scalar is the Goldstone boson of a global broken symmetry. The resulting model is a valid effective theory with a cutoff that can be only a few orders of magnitude below the Planck mass. Moreover, in the khronon case the model has a known candidate UV completion in the form of gravity with anisotropic scaling proposed by P. Hořava.

We have shown that the model exhibits the property of self-acceleration. Namely, in the absence of the cosmological constant and any matter sources the model possesses two solutions corresponding to Minkowski and de Sitter space-times. The former solution is unstable and the presence of an arbitrarily small amount of matter destroys it. The cosmological evolution of a matter-filled universe is driven to the de Sitter attractor. The value of the effective cosmological constant in the de Sitter branch is determined by the lowest dimension coupling between the Goldstone field and the aether. Importantly, it is technically natural to assume this coupling to be small as it is protected from radiative corrections by a discrete symmetry. Thus if one were able to enforce somehow the vanishing of the vacuum energy and the existence of an (unstable) Minkowski vacuum, the value of the current cosmic acceleration would not present fine-tuning problems. However, we left the discussion of a mechanism that could lead to this cancellation of the vacuum energy outside the scope of this article.

We analyzed the phenomenological consequences of the dark energy model proposed in this paper. To this aim, we supplemented the model with cold matter and radiation components. This particular realization was called $\Theta C D M$. We showed that, barring finetuned initial conditions, the homogeneous cosmological evolution in the model is the same as in the universe with a cosmological constant. In other words, the effective dark energy equation of state is $w=-1$. On the other hand, the non-trivial dynamics of dark energy reveals itself in the evolution of cosmological perturbations. We studied this evolution both analytically and numerically in the linear regime and found that the growth of perturbations is enhanced compared to the standard $\Lambda$ CDM case. The enhancement is most prominent at very large scales of order a few gigaparsecs, but extends also to shorter scales. Another property of the model is the appearance of an effective anisotropic stress that leads at very large scales to the difference between the two gravitational potentials in the Newton gauge. In 
principle, the previous effects can allow to discriminate $\Theta C D M$ from $\Lambda$ CDM. Several groups have recently suggested a number of data analysis techniques to test the nature of dark energy and its possible deviations from the cosmological constant [36, 37, 38, 39, 40, 41, 42, 43, 44]. It would be interesting to apply these techniques to the present case and work out the constraints on the parameters of the model following from the present data as well as their expected improvement by future experiments.

Throughout the paper we have assumed a standard Lorentz invariant matter sector. For the Standard Model fields this follows from an overwhelming experimental evidence. However, for the case of dark matter one could relax this assumption and allow it to interact directly with the Lorentz breaking fields. It would be interesting to study how these interactions may affect the evolution of cosmological perturbations. Potentially, this will allow to set bounds on the violation of Lorentz invariance in the dark matter sector. We leave this investigation for the future.

Acknowledgments We are grateful to Eugeny Babichev, Sergei Dubovsky, Jaume Garriga, Dmitry Gorbunov, Ben Hoyle, Ted Jacobson, Julien Lesgourgues, Dmitry Levkov, Shinji Mukohyama, Jorge Noreña, Oriol Pujolàs, Valery Rubakov and Alex Vikman for illuminating discussions. We also thank the organizers and participants of the workshop "Unsolved problems in astrophysics and cosmology" for the stimulating atmosphere during this meeting. D.B. would like to thank the Institute for Nuclear Research of the Russian Academy of Sciences for its warm hospitality. This work was supported in part by the Swiss Science Foundation (D.B.), the Russian Ministry of Education and Science under the state contract 02.740.11.0244 (S.S.), the Grants of the President of Russian Federation NS-5525.2010.2 and MK-3344.2011.2 (S.S.) and the RFBR grants 11-02-92108, 11-02-01528 (S.S.).

\section{A The numerical procedure}

The complete set of equations for the cosmological perturbations is provided by the Einstein's equations (40), equations (411) for the khronon and Goldstone perturbations and the hydrodynamical equations (42) for matter (supplemented by the equation of state for each component). These equations are not independent. For the numerical solution we choose two out of the four Einstein's equations: Eqs. (40c) and (40d). As indicated in the main text, we consider matter consisting of two components: radiation and cold matter. Only the former contributes into the pressure perturbation term in (40c). This implies that to close 
the system it suffices to consider the hydrodynamic equations (42) only for the radiation component. Introducing

$$
\delta_{[\gamma]} \equiv \frac{\delta \rho_{[\gamma]}}{\bar{\rho}_{[\gamma]}}
$$

these equations can be written as a single second order equation,

$$
\ddot{\delta}_{[\gamma]}-\frac{\Delta \delta_{[\gamma]}}{3}-\frac{4 \Delta \phi}{3}-4 \ddot{\psi}=0 .
$$

Performing the Fourier decomposition, normalizing the present scale factor to one, $a\left(t_{0}\right)=1$, and choosing the units such that the present Hubble parameter is equal to one, $H_{0}=1$, we obtain the final system of ordinary differential equations to be solved numerically:

$$
\begin{aligned}
& \ddot{\psi}+\mathcal{H}(\dot{\phi}+2 \dot{\psi})+\left(2 \dot{\mathcal{H}}+\mathcal{H}^{2}\right) \phi-\frac{\beta+\lambda}{2+\alpha B} k^{2}(\dot{\chi}+2 \mathcal{H} \chi)-\frac{\Omega_{\gamma}}{2 a^{2}} \delta_{[\gamma]}=0, \\
& \phi-\psi-\beta(\dot{\chi}+2 \mathcal{H} \chi)=0, \\
& \ddot{\chi}+2 \mathcal{H} \dot{\chi}+\left[c_{\chi}^{2} k^{2}+\dot{\mathcal{H}}(1-B)+\mathcal{H}^{2}(1+B)+a^{2} c_{\Theta}^{4} k_{c}^{2}\right] \chi+a c_{\Theta}^{2} k_{c}^{2} \tilde{\xi} \\
& \ddot{\tilde{\xi}}+2 \mathcal{H} \dot{\tilde{\xi}}+c_{\Theta}^{2} k^{2} \tilde{\xi}+a c_{\Theta}^{4} k^{2} \chi=-a c_{\Theta}^{2} \dot{\phi}-3 a \mathcal{H} c_{\Theta}^{2} \phi, \\
& \ddot{\delta}_{[\gamma]}+\frac{k^{2}}{3} \delta_{[\gamma]}+\frac{4 k^{2}}{3} \phi-4 \ddot{\psi}=0 .
\end{aligned}
$$

Here we have introduced the present radiation density fraction $\Omega_{\gamma}$ and defined $\tilde{\xi}=\xi / \mu^{2}$.

A subtle point is the proper choice of initial conditions for the system (66). These are fixed deep inside the radiation-domination stage when the modes are superhorizon. We consider the initial conditions corresponding to the adiabatic mode. The latter is regular at $t \rightarrow 0$. Thus we write for small $t$ :

$$
\begin{gathered}
\phi=\phi^{(0)}+\phi^{(1)} t, \quad \psi=\psi^{(0)}+\psi^{(1)} t, \quad \delta_{[\gamma]}=\delta_{[\gamma]}^{(0)}+\delta_{[\gamma]}^{(1)} t, \\
\chi=\chi^{(0)} t+\chi^{(1)} \frac{t^{2}}{2}, \quad \tilde{\xi}=\tilde{\xi}^{(0)} \frac{t^{2}}{2}+\tilde{\xi}^{(1)} \frac{t^{3}}{6} .
\end{gathered}
$$

Expanding Eqs. (66) at $t \rightarrow 0$ we obtain the relations:

$$
\begin{gathered}
\delta_{[\gamma]}^{(0)}=-2 \phi^{(0)}, \quad \delta_{[\gamma]}^{(1)}=4 \psi^{(1)}, \\
\phi^{(0)}-\psi^{(0)}-3 \beta \chi^{(0)}=0, \quad \phi^{(1)}-\psi^{(1)}-2 \beta \chi^{(1)}-\frac{\beta \Omega_{c m}}{4 \sqrt{\Omega_{\gamma}}} \phi^{(0)}=0, \\
\chi^{(0)}=\phi^{(0)} / 2, \quad(3+B) \chi^{(1)}=(2+B) \phi^{(1)}+B \psi^{(1)}-\frac{\Omega_{c m}}{4 \sqrt{\Omega_{\gamma}}} \phi^{(0)}, \\
\tilde{\xi}^{(0)}=-\sqrt{\Omega_{\gamma}} c_{\Theta}^{2} \phi^{(0)}, \quad \tilde{\xi}^{(1)}=-2 \sqrt{\Omega_{\gamma}} c_{\Theta}^{2} \phi^{(1)}-\frac{\Omega_{c m}}{2} c_{\Theta}^{2} \phi^{(0)} .
\end{gathered}
$$


Here we have expanded the time dependence of the scale factor at the radiation domination epoch up to the subleading order,

$$
a=\sqrt{\Omega_{\gamma}} t+\frac{\Omega_{c m}}{4} t^{2} .
$$

Additionally, the initial data must satisfy the constraint following from the (00) Einstein's equation 18 (40a). This gives,

$$
\psi^{(1)}+\phi^{(1)}+\frac{\delta_{[\gamma]}^{(1)}}{2}+\frac{\Omega_{c m}}{\sqrt{\Omega_{\gamma}}}\left(\phi^{(0)}+\frac{\delta^{(0)}}{2}\right)=0,
$$

where $\delta$ is the density contrast of cold matter, Eq. (65). For the adiabatic mode the density contrasts are related:

$$
\delta=\frac{3}{4} \delta_{[\gamma]}
$$

Using this relation we obtain that all the coefficients in (67) are determined in terms of $\phi^{(0)}$.

Similar reasoning also gives the equations and initial conditions for $\Lambda$ CDM. As we are interested in the ratios of various quantities in the $\Theta \mathrm{CDM}$ and $\Lambda \mathrm{CDM}$ models we can choose arbitrary normalization for $\phi^{(0)}$, the only requirement being that this normalization is the same for the computations in both model 19 . In practice we choose $\phi^{(0)}=1$.

\section{References}

[1] R. Durrer, "What do we really know about Dark Energy?," arXiv:1103.5331 [astroph.CO].

[2] S. Weinberg, Rev. Mod. Phys. 61, 1 (1989).

[3] G. D. Starkman and R. Trotta, Phys. Rev. Lett. 97, 201301 (2006) arXiv:astro-ph/0607227].

I. Maor, L. Krauss and G. Starkman, Phys. Rev. Lett. 100, 041301 (2008) arXiv:0709.0502 [hep-th]].

\footnotetext{
${ }^{18}$ The $(0 i)$ equation (40b) determines the velocity field and does not impose any constraints.

${ }^{19}$ Strictly speaking, the initial spectrum in the $\Theta C D M$ model may be different from that in $\Lambda$ CDM. Its rigorous determination would require the analysis of the generation of primordial perturbations at the inflationary epoch together with a model for the origin of the Goldstone field. These issues are beyond the scope of the present article. The approximation of identical primordial spectra is enough for our purpose which is to illustrate the difference in the evolution of the cosmological perturbations in the $\Theta C D M$ and $\Lambda \mathrm{CDM}$ models at the late cosmological epoch.
} 
[4] J. B. Hartle and M. Srednicki, Phys. Rev. D 75, 123523 (2007) arXiv:0704.2630 [hepth]]; Phys. Rev. D 81, 123524 (2010) [arXiv:0906.0042 [hep-th]].

[5] D. N. Page, "Typicality Defended," arXiv:0707.4169 [hep-th]; Phys. Lett. B 678, 41 (2009) arXiv:0808.0722 [hep-th]].

[6] J. Garriga and A. Vilenkin, Phys. Rev. D 77 (2008) 043526 [arXiv:0711.2559 [hep-th]].

[7] R. Bousso and R. Harnik, Phys. Rev. D 82 (2010) 123523 [arXiv:1001.1155 [hep-th]].

[8] A. D. Linde, Phys. Lett. B 200, 272 (1988).

[9] S. W. Hawking, Phys. Lett. B 134, 403 (1984).

[10] S. R. Coleman, Nucl. Phys. B 310, 643 (1988).

[11] E. J. Copeland, M. Sami and S. Tsujikawa, Int. J. Mod. Phys. D 15, 1753 (2006) arXiv:hep-th/0603057.

[12] J. A. Frieman, C. T. Hill, A. Stebbins, I. Waga, Phys. Rev. Lett. 75, 2077-2080 (1995). astro-ph/9505060.

[13] R. Kallosh, A. D. Linde, D. A. Linde and L. Susskind, Phys. Rev. D 52 (1995) 912 arXiv:hep-th/9502069.

[14] P. Hořava, Phys. Rev. D 79 (2009) 084008 [arXiv:0901.3775 [hep-th]].

[15] D. Blas, O. Pujolas and S. Sibiryakov, Phys. Rev. Lett. 104, 181302 (2010) arXiv:0909.3525 [hep-th]].

[16] D. Blas, O. Pujolas and S. Sibiryakov, Phys. Lett. B 688, 350 (2010) arXiv:0912.0550 [hep-th]].

[17] D. Blas, O. Pujolas and S. Sibiryakov, JHEP 1104, 018 (2011) arXiv:1007.3503 [hepth]].

[18] T. Jacobson, Phys. Rev. D 81, 101502 (2010) [Erratum-ibid. D 82, 129901 (2010)] arXiv:1001.4823 [hep-th]].

[19] T. Jacobson and D. Mattingly, Phys. Rev. D 64, 024028 (2001) arXiv:gr-qc/0007031. 
[20] N. Arkani-Hamed, H. C. Cheng, M. A. Luty and S. Mukohyama, JHEP 0405 (2004) 074 [arXiv:hep-th/0312099].

[21] V. A. Kostelecky and N. Russell, Rev. Mod. Phys. 83, 11 (2011) arXiv:0801.0287 [hep$\mathrm{ph}]$.

[22] S. Groot Nibbelink and M. Pospelov, Phys. Rev. Lett. 94, 081601 (2005) arXiv:hep-ph/0404271.

[23] P. A. Bolokhov, S. G. Nibbelink and M. Pospelov, Phys. Rev. D 72, 015013 (2005) arXiv:hep-ph/0505029.

[24] G. F. Giudice, M. Raidal and A. Strumia, Phys. Lett. B 690, 272 (2010) arXiv:1003.2364 [hep-ph]].

[25] D. S. Gorbunov and S. M. Sibiryakov, "Self-accelerated brane Universe with warped extra dimension," arXiv:0804.2248 [hep-th].

[26] C. M. Will, Living Rev. Rel. 9, 3 (2005) arXiv:gr-qc/0510072.

[27] S. M. Carroll and E. A. Lim, Phys. Rev. D 70, 123525 (2004) arXiv:hep-th/0407149].

[28] D. Blas, H. Sanctuary, Phys. Rev. D84, 064004 (2011) [arXiv:1105.5149 [gr-qc]].

[29] T. Jacobson, PoS QG-PH, 020 (2007) arXiv:0801.1547 [gr-qc]].

[30] M. Nakashima and T. Kobayashi, "The Music of the Aetherwave - B-mode Polarization in Einstein-Aether Theory," arXiv:1103.2197 [astro-ph.CO].

[31] W. Donnelly, T. Jacobson, Phys. Rev. D82, 064032 (2010) arXiv:1007.2594 [gr-qc]].

[32] M. Libanov, V. Rubakov, E. Papantonopoulos, M. Sami and S. Tsujikawa, JCAP 0708, 010 (2007) arXiv:0704.1848 [hep-th]].

[33] D. Blas, S. Sibiryakov, in progress.

[34] T. Kobayashi, Y. Urakawa and M. Yamaguchi, JCAP 1004, 025 (2010) arXiv:1002.3101 [hep-th]].

[35] C. Armendariz-Picon, N. F. Sierra and J. Garriga, JCAP 1007, 010 (2010) arXiv:1003.1283 [astro-ph.CO]]. 
[36] L. Amendola, M. Kunz and D. Sapone, JCAP 0804 (2008) 013 arXiv:0704.2421 [astro$\mathrm{ph}]]$.

[37] Y. -S. Song, L. Hollenstein, G. Caldera-Cabral, K. Koyama, JCAP 1004 (2010) 018. arXiv:1001.0969 [astro-ph.CO]].

[38] S. F. Daniel, E. V. Linder, T. L. Smith, R. R. Caldwell, A. Cooray, A. Leauthaud, L. Lombriser, Phys. Rev. D81 (2010) 123508. [arXiv:1002.1962 [astro-ph.CO]].

[39] L. Pogosian, A. Silvestri, K. Koyama, G. -B. Zhao, Phys. Rev. D81 (2010) 104023. arXiv:1002.2382 [astro-ph.CO]].

[40] R. Bean, M. Tangmatitham, Phys. Rev. D81 (2010) 083534. arXiv:1002.4197 [astroph.CO]].

[41] C. Shapiro, S. Dodelson, B. Hoyle, L. Samushia and B. Flaugher, Phys. Rev. D 82 (2010) 043520 arXiv:1004.4810 [astro-ph.CO]].

[42] D. E. Holz, S. Perlmutter, "The most massive objects in the Universe," arXiv:1004.5349 [astro-ph.CO]].

[43] M. J. Mortonson, W. Hu, D. Huterer, Phys. Rev. D83 (2011) 023015. arXiv:1011.0004 [astro-ph.CO]].

[44] Y. -S. Song, G. -B. Zhao, D. Bacon, K. Koyama, R. C. Nichol, L. Pogosian, arXiv:1011.2106 [astro-ph.CO]]. 